\title{
ANALYSIS OF FINITE ELEMENT DISCRETIZATIONS OF AN OPTIMAL CONTROL FORMULATION OF THE IMAGE REGISTRATION PROBLEM*
}

\author{
EUNJUNG LEE $^{\dagger}$ AND MAX GUNZBURGER ${ }^{\ddagger}$
}

\begin{abstract}
The goal of image registration is to determine a transformation of an image so that the resulting image is close to another image. We use an optimal control approach to determining the transformation. We precisely define the optimal control problem that solves the image registration problem, and state the optimality system corresponding to that control problem. We then define finite element discretizations of the optimality system and derive error estimates for the approximate solutions. We also define and prove the convergence of a gradient iterative method for the solution of the discrete optimality system.
\end{abstract}

Key words. Image registration, finite element methods, optimal control, gradient methods.

AMS subject classifications. 65D18, 94A08, 49M05, 35Q93, 65N30

1. Introduction. Image registration is one of the fundamental tasks in image processing [15] and is widely used in various applications [1, 8, 11, 14]. Given two images $\mathbf{R}(\mathbf{x})$ and $\mathbf{T}(\mathbf{x})$, referred to as the reference and template images, respectively, the goal of image registration is to find a reasonable geometric transformation which minimizes the dissimilarity between the reference image and the transformed template image. This paper continues the work in paper [12] in which the grid deformation method is used to define an optimal control formulation for the image registration problem. Then, the Lagrange multiplier rule is used to derive an optimality system, i.e., a system of partial differential equations, whose solution yields the desired transformation.

The grid deformation method constructs a vector field $\mathbf{u}$ that satisfies a div-curl system of the form

$$
\begin{cases}\nabla \cdot \mathbf{u}=f-1 & \text { in the image domain } \\ \nabla \times \mathbf{u}=g & \text { in the image domain } \\ \mathbf{n} \cdot \mathbf{u}=0 & \text { on the boundary of the image domain. }\end{cases}
$$

The vector field $\mathbf{u}$ is used to generate a transformation $\phi$ to move the grid in a desired way by solving the nonlinear ordinary differential equation

$$
\left\{\begin{array}{l}
\frac{\partial}{\partial t} \boldsymbol{\phi}(t, \mathbf{x})=\mathbf{u}(t, \phi(t, \mathbf{x})) \quad 0<t \leq 1 \\
\phi(0, \mathbf{x})=\mathbf{x}
\end{array}\right.
$$

at every point in the image domain.

Once the transformation $\phi(t, \mathbf{x})$ is obtained, one use it to evaluate some measure of the difference between the transformed template image $\mathbf{T}(\phi(1, \mathbf{x}))$ and the reference image $\mathbf{R}(\mathbf{x})$. Our goal is to determine right-hand side "controls" $f$ and $g$ so that the dissimilarity measure between the reference and transformed template images is made

\footnotetext{
*Supported in part by the U.S. National Science Foundation under grant DMS0932948.

${ }^{\dagger}$ Department of Computational Science and Engineering, Yonsei University, Seoul 120-749, Korea (nuejlee@gmail.com).

${ }^{\ddagger}$ Department of Scientific Computing, Florida State University, Tallahassee FL 32306-4120 (gunzburg@fsu.edu).
} 
as small as possible. To this end, in [12], an optimal control problem is formulated in which a dissimilarity measure is defined and then minimized with respect to $f$ and $g$ in appropriate function spaces, with the differential equations (1.1) and (1.2) acting as constraints. The existence of optimal solutions is proven, as is the existence of suitable Lagrange multipliers that are used to convert the constrained optimization problem into an unconstrained one. In addition, and optimality system consisting of (1.1) and (1.2), adjoint equations for the Lagrange multiplier variables, and optimality conditions for the controls $f$ and $g$ are rigorously derived.

In Section 2, we precisely define the optimal control problem that solves the image registration problem and state the optimality system corresponding to that control problem. For details, see [12]. Then, in Section 3, we define finite element discretizations of the optimality system and derive error estimates for the approximate solutions; first, in Section 3.1, we separately consider the components of the optimality system and then, in Section 3.2, we put it all together to derive error estimates for the fully-coupled discretized optimality system. Solving the coupled discretized optimality system is a formidable task so that, in Section 4, we also define a gradient iterative method for its solution in which the different components of the optimality system, i.e., the state and adjoint equations and the optimality conditions, are solved sequentially at each iteration. We also prove, under suitable hypotheses, that the gradient method converges. In this paper, we do not provide computational examples because several such examples are already provided in [12].

2. Optimal control problem. In [12], an optimal control problem for image registration was introduced and analyzed; the approach is based on the grid deformation method $[3,13,16]$ and seeks to minimize an objective functional that measures the difference between the transformed image and the reference image. The existence of an optimal transformation is proved as is the applicability of the Lagrange multiplier method. Then, an optimality system from which optimal transformations can be obtained is derived. In this section, we recall the results derived in [12].

Let $\Omega \subset \mathbb{R}^{2}$ denote a bounded domain that is a convex polygon or that has a $\mathcal{C}^{1,1}$ boundary $\Gamma$; usually, $\Omega$ is a rectangle. Let $\|\cdot\|$ denote the $L^{2}(\Omega)$-norm, $\|\cdot\|_{\infty}$ the $L^{\infty}(\Omega)$-norm, and $\|\cdot\|_{m}$ the standard norm in the Sobolev space $H^{m}(\Omega)$. For domains other than $\Omega$, we explicitly indicate the domain in the norm notation; for example, $\|\cdot\|_{L^{2}(\tau)}$ denotes the $L^{2}(\tau)$-norm. Let $\|\cdot\|_{m, \infty}$ denote the standard norm for the Sobolev space $W_{\infty}^{m}(\Omega)=\left\{u: D^{\alpha} u \in L^{\infty}(\Omega), 0 \leq|\alpha| \leq m\right\}$. Let $\langle\cdot, \cdot\rangle$ denote the $L^{2}(\Omega)$ inner product or the duality pairing based with $L^{2}(\Omega)$ acting as the pivot space. We use the same norm and inner product notations for spaces of vector-valued functions. Also, we have the scalar curl operator $\nabla \times \mathbf{u}=\partial_{x} u_{2}-\partial_{y} u_{1}$ and the vectorcurl operator $\nabla^{\perp} u=\left(\partial_{y} u,-\partial_{x} u\right)^{t}$. Assume that the reference image $\mathbf{R}(\mathbf{x})$ and the template image $\mathbf{T}(\mathbf{x})$ belong to at least $\left[H^{2+\delta}(\Omega)\right]^{2}$ with $\delta>0$.

We define the subspaces and product Hilbert spaces

$$
\begin{gathered}
H_{N}^{1}(\Omega)=\left\{\mathbf{u} \in\left[H^{1}(\Omega)\right]^{2}: \mathbf{n} \cdot \mathbf{u}=0 \text { on } \Gamma\right\} \\
H_{D}^{1}\left(\Omega=\left\{\mathbf{u} \in\left[H^{1}(\Omega)\right]^{2}: \mathbf{n} \times \mathbf{u}=\mathbf{0} \text { on } \Gamma\right\}\right. \\
\Lambda=\left(\left[H^{2}(\Omega)\right]^{2} \cap H_{N}^{1}(\Omega)\right) \times\left[H^{1}\left((0,1) ; L^{2}(\Omega)\right)\right]^{2} \times H^{1}(\Omega) \times H^{1}(\Omega) \\
\Theta=H^{1}(\Omega) \times H^{1}(\Omega) \times\left[L^{2}((0,1) \times \Omega)\right]^{2} \times \mathbb{R} \times H^{\frac{3}{2}}(\Gamma) \times L^{2}(\Omega)
\end{gathered}
$$

and then define the operator $M: \Lambda \mapsto \Theta$ as follows:

$$
M(\mathbf{u}, \phi, f, g)=0
$$


if and only if

$$
\begin{array}{rlrl}
\langle\nabla \cdot \mathbf{u}-f+1, \xi\rangle=0 & & \forall \xi \in H^{-1}(\Omega) \\
\langle\nabla \times \mathbf{u}-g, \eta\rangle=0 & & \forall \eta \in H^{-1}(\Omega) \\
\left\langle\frac{\partial \phi}{\partial t}-\mathbf{u}(\boldsymbol{\phi}), \boldsymbol{\psi}\right\rangle=0 & & \forall \boldsymbol{\psi} \in\left[L^{2}((0,1) \times(\Omega))\right]^{2} \\
\sigma \int_{\Omega}(f-1) d \mathbf{x}=0 & & \forall \sigma \in \mathbb{R} \\
\langle\mathbf{n} \cdot \mathbf{u}, \nu\rangle=0 & \forall \nu \in L^{2}\left((0,1) ; H^{-\frac{3}{2}}(\Gamma)\right) \\
\langle\boldsymbol{\phi}(0, \mathbf{x})-\mathbf{x}, \boldsymbol{\mu}\rangle=0 & \forall \boldsymbol{\mu} \in\left[L^{2}(\Omega)\right]^{2} .
\end{array}
$$

Now, we define the objective functional

$$
\begin{aligned}
& J\left(\left.\boldsymbol{\phi}\right|_{t=1}, f, g\right)=\frac{1}{2}\|\mathbf{T}(\phi(1, \mathbf{x}))-\mathbf{R}\|^{2} \\
& \quad+\frac{\alpha_{f_{0}}}{2}\|f\|^{2}+\frac{\alpha_{f_{1}}}{2}\|\nabla f\|^{2}+\frac{\alpha_{g_{0}}}{2}\|g\|^{2}+\frac{\alpha_{g_{1}}}{2}\|\nabla g\|^{2}-\beta \int_{\Omega} \log f d \mathbf{x},
\end{aligned}
$$

where $\alpha_{f_{0}}, \alpha_{f_{1}}, \alpha_{g_{0}}$, and $\alpha_{g_{1}}$ are penalty parameters and $\beta$ is a barrier parameter. The first term is the object of optimization, i.e., finding a transformation $\phi(1, \mathbf{x})$ such that the transformed template image $\mathbf{T}(\boldsymbol{\phi})$ is a "close" as possible to the reference image R. The penalty terms are used to implicitly limit the size of the "controls" $f$ and $g$ whereas the barrier term ensures that the additional constraing $f>0$ is satisfied. See [12] for details about the problem formulation.

The set of all admissible solutions is defined by

$$
A_{a d}=\left\{(\mathbf{u}, \phi, f, g) \in V \mid J\left(\left.\phi\right|_{t=1}, f, g\right) \text { is bounded and (2.1) is satisfied }\right\} .
$$

Then, the optimal control problem for image registration is given by:

find $(\mathbf{u}, \phi, f, g) \in A_{a d}$ that minimizes the functional (2.2).

In [12], it is proved that an optimal solution exists as does a suitable Lagrange multiplier that turns the constrained optimization problem (2.3) into an unconstrained problem. In addition, a corresponding optimality system is rigorously derived. We have that the optimal state $(\mathbf{u}, \phi) \in H^{2}(\Omega) \times L^{2}\left(H^{1}(I) ; \Omega\right)$, the optimal control $(f, g) \in H^{1}(\Omega) \times H^{1}(\Omega)$, and the corresponding Lagrange multiplier $(\xi, \eta, \boldsymbol{\psi}, \sigma, \nu, \boldsymbol{\mu}) \in$ $H^{-1}(\Omega) \times H^{-1}(\Omega) \times L^{2}(I ; \Omega) \times \mathbb{R} \times L^{2}\left(I ; H^{-\frac{3}{2}}(\Gamma)\right) \times L^{2}(\Omega)$. The Lagrangian functional is defined as

$$
\begin{aligned}
& L(\mathbf{u}, \boldsymbol{\phi}, f, g ; \xi, \eta, \boldsymbol{\psi}, \sigma, \nu, \boldsymbol{\mu}) \\
& =J\left(\left.\boldsymbol{\phi}\right|_{t=1}, f, g\right)-\int_{\Omega}(\nabla \cdot \mathbf{u}-f+1) \xi d \mathbf{x}-\int_{\Omega}(\nabla \times \mathbf{u}-g) \eta d \mathbf{x} \\
& \quad-\int_{\Omega} \int_{I}\left(\frac{\partial \phi}{\partial t}-\mathbf{u}(t, \phi)\right) \cdot \boldsymbol{\psi} d t d \mathbf{x}-\sigma \int_{\Omega} f-1 d \mathbf{x} \\
& \quad-\int_{\Gamma} \int_{I}(\mathbf{n} \cdot \mathbf{u}) \nu d t d \mathbf{x}-\int_{\Omega}(\phi(0, \mathbf{x})-\mathbf{x}) \cdot \boldsymbol{\mu} d \mathbf{x} .
\end{aligned}
$$

The optimality system consists of the state system

$$
\begin{cases}\nabla \cdot \mathbf{u}=f-1 & \text { in } \Omega \\ \nabla \times \mathbf{u}=g & \text { in } \Omega \\ \mathbf{n} \cdot \mathbf{u}=0 & \text { on } \partial \Omega\end{cases}
$$


and

$$
\begin{cases}\frac{\partial \phi}{\partial t}=\mathbf{u}(\phi) & \text { in } I \times \Omega \\ \phi(0, \mathbf{x})=\mathbf{x} & \text { in } \Omega\end{cases}
$$

the adjoint system

$$
\begin{cases}\frac{\partial \boldsymbol{\psi}}{\partial t}+\nabla_{\boldsymbol{\phi}} \mathbf{u}(\boldsymbol{\phi}) \boldsymbol{\psi}=0 & \text { in } I \times \Omega \\ \boldsymbol{\psi}(1, \mathbf{x})=(T(\phi(1, \mathbf{x}))-R(\mathbf{x})) \cdot \nabla_{\boldsymbol{\phi}} T(\boldsymbol{\phi}(1, \mathbf{x})) & \text { in } \Omega\end{cases}
$$

and

$$
\begin{cases}\nabla^{\perp} \eta-\nabla \xi=\int_{I}\left|\nabla \phi^{-1}(t, \mathbf{x})\right| \psi\left(t, \phi^{-1}(t, \mathbf{x})\right) d t & \text { in } \Omega \\ \eta=0 & \text { on } \Gamma\end{cases}
$$

and the optimality conditions

$$
\left\{\begin{aligned}
-\alpha_{f_{1}} \Delta f+\alpha_{f_{0}} f=\sigma-\xi & \text { in } \Omega \\
-\alpha_{g_{1}} \Delta g+\alpha_{g_{0}} g=-\eta & \text { in } \Omega \\
\mathbf{n} \cdot \nabla f=0 & \text { on } \Gamma \\
g=0 & \text { on } \Gamma,
\end{aligned}\right.
$$

where $\sigma$ is defined by integrating both sides of the first equation in (2.8):

$$
\sigma=\alpha_{f_{0}}+\frac{1}{|\Omega|} \int_{\Omega} \xi d \mathbf{x} .
$$

Here, the Lagrange multiplier pair $(\nu, \boldsymbol{\mu})$ is omitted since it does not play any role in determining the optimal state, control, and other Lagrange multipliers.

In Section 3, we derive error estimates for finite element approximations of the solution of the optimality system (2.4)-(2.8) and then, in Section 4, we discuss how to solve the discretized equations.

3. Finite element discretizations of the optimality system. Let $\mathcal{T}_{h}$ be a partition of the domain $\bar{\Omega}=\cup_{\tau \in \mathcal{T}_{h}} \tau$ into finite elements $\tau \in \mathcal{T}_{h}$. Let $h:=\max \left\{h_{\tau}:=\right.$ $\left.\operatorname{diam}(\tau): \tau \in \mathcal{T}_{h}\right\}$. Assume that the partition $\mathcal{T}_{h}$ is regular so that we can construct a conforming finite element space that satisfies standard approximation properties; see [7]. We also assume that there exists a constant $\rho$ such that $h \leq \rho h_{\tau}$ for all $\tau \in \mathcal{T}_{h}$. Let $P_{k}$ denote the space of all polynomials of degree $\leq k$ with respect to each variable.

The next result is useful in several subsequent proofs.

Lemma 3.1. Let $(\mathbf{u}, \phi, f, g)$ denote a solution of the optimality system (2.4)-(2.8) belonging to the admissibility set $A_{\text {ad }}$ so that $\|f\|_{1},\|g\|_{1}$, and $\|\mathbf{u}\|_{2}$ are bounded. Then, $\mathbf{u} \in W_{\infty}^{2}(\Omega)$, i.e., there exists a constant $M_{u}$ such that

$$
\left|D^{\alpha} \mathbf{u}(\mathbf{x})\right| \leq M_{u} \quad \text { for } 0 \leq|\alpha| \leq 2 \quad \text { a.e. in } \Omega \text {. }
$$

Proof. Suppose $\mathbf{u} \notin W_{\infty}^{2}(\Omega)$. Then, there exists a set $U \subset \Omega$ such that $m(U)$, the measure of $U$, is not 0 and $\left|D^{\alpha} \mathbf{u}(\mathbf{x})\right| \geq n$ for some $0 \leq|\alpha| \leq 2$ and for all $\mathbf{x} \in U$. Then,

$$
\int_{\Omega} \sum_{0 \leq|\alpha| \leq 2}\left|D^{\alpha} \mathbf{u}(\mathbf{x})\right|^{2} d \mathbf{x} \geq \int_{U} \sum_{0 \leq|\alpha| \leq 2}\left|D^{\alpha} \mathbf{u}(\mathbf{x})\right|^{2} d \mathbf{x} \geq n^{2} \cdot m(U) \longrightarrow \infty
$$


as $n \rightarrow \infty$, which contradicts the fact $\|\mathbf{u}\|_{2}$ is bounded.

This section is composed of two parts. In Section 3.1, we present several error estimates for the solutions of discretizations of the individual components of the optimality system (2.4)-(2.8). Then, in Section 3.2, we define a fully discretized optimality system and show the convergence of approximate solutions of that system, using the results obtained in Section 3.1.

3.1. Optimal error estimates for the components of the optimality system. We denote by $(\mathbf{u}, \boldsymbol{\phi}, f, g, \boldsymbol{\psi}, \xi, \eta)$ a solution of the optimality system (2.4)-(2.8).

3.1.1. Error estimates for (2.4). We first use a least-squares finite element method to solve the system (2.4) in the case where the exact controls $f$ and $g$ are assumed known. To this end, we minimize the least-squares residual functional

$$
\mathcal{F}(\mathbf{v} ; f, g)=\|\nabla \cdot \mathbf{v}-f+1\|^{2}+\|\nabla \times \mathbf{v}-g\|^{2}
$$

by solving the corresponding first-order necessary conditions: for given $f, g \in H^{1}(\Omega)$, determine $\mathbf{u} \in H_{N}^{1}(\Omega)$ such that

$$
\langle\nabla \cdot \mathbf{u}, \nabla \cdot \mathbf{v}\rangle+\langle\nabla \times \mathbf{u}, \nabla \times \mathbf{v}\rangle=\langle f-1, \nabla \cdot \mathbf{v}\rangle+\langle g, \nabla \times \mathbf{v}\rangle
$$

for all $\mathbf{v} \in H_{N}^{1}(\Omega)$. Note that it is known that the solution of this problem is actually more regular, i.e., we have $\mathbf{u} \in\left[H^{2}(\Omega)\right]^{2} \cap H_{N}^{1}(\Omega)$.

Next, define the finite-dimensional subspace

$$
\mathcal{U}^{h}=\left\{\mathbf{u} \in \mathcal{C}^{0}(\Omega):\left.\mathbf{u}\right|_{\tau} \in P_{k}(\tau), k=1,2\right\} \cap H_{N}^{1}(\Omega) \subset H_{N}^{1}(\Omega)
$$

and then pose the discrete least-squares problem

$$
\widehat{\mathbf{u}}^{h}=\arg \min _{\mathbf{v}^{h} \in \mathcal{U}^{h}} \mathcal{F}\left(\mathbf{v}^{h} ; f, g\right)
$$

whose solution can be obtained by determining $\widehat{\mathbf{u}}^{h} \in \mathcal{U}^{h}$ satisfying

$$
\left\langle\nabla \cdot \widehat{\mathbf{u}}^{h}, \nabla \cdot \mathbf{v}^{h}\right\rangle+\left\langle\nabla \times \widehat{\mathbf{u}}^{h}, \nabla \times \mathbf{v}^{h}\right\rangle=\left\langle f-1, \nabla \cdot \mathbf{v}^{h}\right\rangle+\left\langle g, \nabla \times \mathbf{v}^{h}\right\rangle
$$

for all $\mathbf{v}^{h} \in \mathcal{U}^{h}$.

Proposition 3.2. Let $\mathbf{u} \in H^{2}(\Omega) \cap H_{N}^{1}(\Omega)$ and $\widehat{\mathbf{u}}^{h} \in \mathcal{U}^{h}$ satisfy (3.3) and (3.4), respectively. Then,

$$
\left\|\mathbf{u}-\widehat{\mathbf{u}}^{h}\right\|_{r} \leq c h^{2-r}\|\mathbf{u}\|_{2} \quad \text { for } r=0,1
$$

Proof. This a standard result for least-squares finite element methods [2].

3.1.2. Error estimates for (2.5). Let $\left\{t_{n}\right\}_{n=0}^{N}$ be a partition of $[0,1]$ into equal intervals $\Delta t=1 / N$ with $t_{0}=0$ and $t_{N}=1$. Supposing the exact solution $\mathbf{u}$ of $(2.4)$ is known, to determine an approximation $\phi_{N}\left(t_{n}, \cdot\right) \approx \phi\left(t_{n}, \cdot\right)$ of $(2.5)$, we apply the forward Euler method, i.e., at time $t=t_{n+1}$,

$$
\phi_{N}\left(t_{n+1}, \mathbf{x}\right)=\phi_{N}\left(t_{n}, \mathbf{x}\right)+\Delta t \mathbf{u}\left(\phi_{N}\left(t_{n}, \mathbf{x}\right)\right) \quad \text { with } \quad \phi_{N}(0, \mathbf{x})=\mathbf{x}
$$

so that

$$
\left|\phi\left(t_{n+1}, \mathbf{x}\right)-\phi_{N}\left(t_{n+1}, \mathbf{x}\right)\right| \sim O(\Delta t)
$$


We also define an approximation $\widehat{\phi}_{N}^{h}$ of the problem (2.5) in which, instead of the exact solution $\mathbf{u}$, we use the approximate solution $\widehat{\mathbf{u}}^{h}$ from Section 3.1.1. That approximation is determined from

$$
\widehat{\boldsymbol{\phi}}_{N}^{h}\left(t_{n+1}, \mathbf{x}\right)=\widehat{\boldsymbol{\phi}}_{N}^{h}\left(t_{n}, \mathbf{x}\right)+\Delta t \widehat{\mathbf{u}}^{h}\left(\widehat{\boldsymbol{\phi}}_{N}^{h}\left(t_{n}, \mathbf{x}\right)\right) \quad \text { with } \quad \widehat{\boldsymbol{\phi}}_{N}^{h}(0, \mathbf{x})=\mathbf{x} .
$$

Then, $\widehat{\phi}_{N}^{h}\left(t_{n+1}, \cdot\right)$ belongs to the finite-dimensional space

$$
\Phi^{h}=\left\{\phi:\left.\phi\right|_{\tau} \in P_{\widehat{n}}(\tau) \text { for some } \widehat{n} \geq 0, \forall \tau \in \mathcal{T}_{h}\right\} \subset L^{2}(\Omega) .
$$

Our goal is to estimate the error $\left\|\phi-\widehat{\phi}_{N}^{h}\right\|$. By the triangle inequality,

$$
\left\|\phi-\widehat{\phi}_{N}^{h}\right\| \leq\left\|\phi-\phi_{N}\right\|+\left\|\phi_{N}-\widehat{\phi}_{N}^{h}\right\| .
$$

Because of (3.7), we focus on the term $\left\|\phi_{N}-\widehat{\phi}_{N}^{h}\right\|$. For notational simplification, we abbreviate $\boldsymbol{\phi}_{N}\left(t_{n}, \mathbf{x}\right)$ to $\boldsymbol{\phi}_{n}(\mathbf{x})$ and $\widehat{\boldsymbol{\phi}}_{N}^{h}\left(t_{n}, \mathbf{x}\right)$ to $\widehat{\boldsymbol{\phi}}_{n}^{h}(\mathbf{x})$.

We introduce an interpolation error estimate. Let the interpolation operator $\mathrm{I}^{h} \in \mathcal{L}\left(W_{\infty}^{2}(\Omega) \cap H^{2}(\Omega): \mathcal{U}^{h}\right)$, with $\left.\mathrm{I}^{h} v\right|_{\tau} \in P_{k}(\tau)$ on each element $\tau$, satisfy the following error estimates for all integers $r$ and $s$ with $0 \leq r \leq 2$ and $0 \leq s \leq 1$, respectively (see [4, Theorem 4.4.20] for details): for all $v \in W_{\infty}^{2}(\Omega) \cap H^{2}(\Omega)$,

$$
\begin{gathered}
\left(\sum_{\tau \in \mathcal{T}_{h}}\left\|v-\mathrm{I}^{h} v\right\|_{H^{r}(\tau)}^{2}\right)^{\frac{1}{2}} \leq c h^{2-r}\|v\|_{2}, \\
\max _{\tau \in \mathcal{T}_{h}}\left\|v-\mathrm{I}^{h} v\right\|_{W_{\infty}^{s}(\tau)} \leq c h^{1-s}\|v\|_{2} .
\end{gathered}
$$

The following lemma is a well-known inverse estimate [4].

Lemma 3.3. Let $\mathcal{P}$ be a finite-dimensional subspace of $W_{p}^{l}(K) \cap W_{q}^{m}(K)$, where $1 \leq p \leq \infty, 1 \leq q \leq \infty$, and $0 \leq m \leq l$. Then, there exists a constant $c$ such that, for all $v \in \mathcal{P}$

$$
\|v\|_{W_{p}^{l}(K)} \leq c h^{m-l+\frac{d}{p}-\frac{d}{q}}\|v\|_{W_{q}^{m}(K)},
$$

where $d$ is the dimension of $K$.

In the next theorem, we derive the $L^{2}$-error bound for $\phi_{N}-\widehat{\phi}_{N}^{h}$ at a fixed time $t=t_{n}$.

Proposition 3.4. Let $\Delta t$ be sufficiently small. Then, at each fixed time $t=t_{n}$, $n=0, \ldots, N$, we have

$$
\left\|\phi_{n}-\widehat{\phi}_{n}^{h}\right\| \leq c h^{2}\|\mathbf{u}\|_{2, \infty}
$$

Proof. From (3.6) and (3.8), at time $t=t_{n+1}$ we have

$$
\phi_{n+1}(\mathbf{x})-\widehat{\boldsymbol{\phi}}_{n+1}^{h}(\mathbf{x})=\phi_{n}(\mathbf{x})-\widehat{\boldsymbol{\phi}}_{n}^{h}(\mathbf{x})+\Delta t\left(\mathbf{u}\left(\phi_{n}(\mathbf{x})\right)-\widehat{\mathbf{u}}^{h}\left(\widehat{\boldsymbol{\phi}}_{n}^{h}(\mathbf{x})\right)\right) .
$$

First, at time $t=0$, we have $\phi_{0}(\mathbf{x})-\widehat{\phi}_{0}^{h}(\mathbf{x})=\mathbf{x}-\mathbf{x}=\mathbf{0}$. Then, at time $t_{1}$, we have

$$
\phi_{1}(\mathbf{x})-\widehat{\boldsymbol{\phi}}_{1}^{h}(\mathbf{x})=\Delta t\left(\mathbf{u}\left(\phi_{0}(\mathbf{x})\right)-\widehat{\mathbf{u}}^{h}\left(\widehat{\boldsymbol{\phi}}_{0}^{h}(\mathbf{x})\right)\right) .
$$

At time $t=t_{2}$, we have

$$
\begin{aligned}
\boldsymbol{\phi}_{2}(\mathbf{x})-\widehat{\boldsymbol{\phi}}_{2}^{h}(\mathbf{x}) & =\phi_{1}(\mathbf{x})-\widehat{\boldsymbol{\phi}}_{1}^{h}(\mathbf{x})+\Delta t\left(\mathbf{u}\left(\boldsymbol{\phi}_{1}(\mathbf{x})\right)-\widehat{\mathbf{u}}^{h}\left(\widehat{\boldsymbol{\phi}}_{1}^{h}(\mathbf{x})\right)\right) \\
& =\Delta t \sum_{i=0}^{1}\left(\mathbf{u}\left(\boldsymbol{\phi}_{i}(\mathbf{x})\right)-\widehat{\mathbf{u}}^{h}\left(\widehat{\boldsymbol{\phi}}_{i}^{h}(\mathbf{x})\right)\right) .
\end{aligned}
$$


If we repeat the above process inductively, we have

$$
\phi_{n+1}(\mathbf{x})-\widehat{\phi}_{n+1}^{h}(\mathbf{x})=\Delta t \sum_{i=0}^{n}\left(\mathbf{u}\left(\phi_{i}(\mathbf{x})\right)-\widehat{\mathbf{u}}^{h}\left(\widehat{\phi}_{i}^{h}(\mathbf{x})\right)\right) .
$$

By the triangle inequality, we have

$$
\left\|\boldsymbol{\phi}_{n+1}-\widehat{\boldsymbol{\phi}}_{n+1}^{h}\right\|^{2} \leq c \Delta t^{2} \sum_{i=0}^{n}\left(\left\|\mathbf{u}\left(\boldsymbol{\phi}_{i}\right)-\widehat{\mathbf{u}}^{h}\left(\boldsymbol{\phi}_{i}\right)\right\|^{2}+\left\|\widehat{\mathbf{u}}^{h}\left(\boldsymbol{\phi}_{i}\right)-\widehat{\mathbf{u}}^{h}\left(\widehat{\boldsymbol{\phi}}_{i}^{h}\right)\right\|^{2}\right) .
$$

Consider $\widehat{\mathbf{u}}^{h}\left(\boldsymbol{\phi}_{i}\right)-\widehat{\mathbf{u}}^{h}\left(\widehat{\boldsymbol{\phi}}_{i}^{h}\right)$ elementwise from

$$
\left\|\widehat{\mathbf{u}}^{h}\left(\boldsymbol{\phi}_{i}\right)-\widehat{\mathbf{u}}^{h}\left(\widehat{\boldsymbol{\phi}}_{i}^{h}\right)\right\|^{2}=\sum_{\tau \in \mathcal{T}_{h}}\left\|\widehat{\mathbf{u}}^{h}\left(\boldsymbol{\phi}_{i}\right)-\widehat{\mathbf{u}}^{h}\left(\widehat{\boldsymbol{\phi}}_{i}^{h}\right)\right\|_{L^{2}(\tau)}^{2} .
$$

If $\widehat{\mathbf{u}}^{h}$ is piecewise linear, then

$$
\left\|\widehat{\mathbf{u}}^{h}\left(\boldsymbol{\phi}_{i}\right)-\widehat{\mathbf{u}}^{h}\left(\widehat{\boldsymbol{\phi}}_{i}^{h}\right)\right\|_{L^{2}(\tau)}^{2} \leq c\left\|\widehat{\mathbf{u}}^{h}\right\|_{\infty}^{2}\left\|\phi_{i}-\widehat{\boldsymbol{\phi}}_{i}^{h}\right\|_{L^{2}(\tau)}^{2} .
$$

If $\widehat{\mathbf{u}}^{h}$ is piecewise quadratic, instead of (3.14), some calculations yield

$$
\left\|\widehat{\mathbf{u}}^{h}\left(\boldsymbol{\phi}_{i}\right)-\widehat{\mathbf{u}}^{h}\left(\widehat{\boldsymbol{\phi}}_{i}^{h}\right)\right\|_{L^{2}(\tau)}^{2} \leq c\left\|\nabla \widehat{\mathbf{u}}^{h}\right\|_{\infty}^{2}\left\|\boldsymbol{\phi}_{i}-\widehat{\boldsymbol{\phi}}_{i}^{h}\right\|_{L^{2}(\tau)}^{2} .
$$

Recall the interpolation operator $\mathrm{I}^{h}$ satisfying (3.9) and (3.10). Then, the triangle inequality, the inverse inequality (3.11) (with $l=m=0, p=\infty, q=2$ ), the regularity result (3.5), (3.9), (3.10), and the Sobolev imbedding theorem yield

$$
\begin{aligned}
\left\|\widehat{\mathbf{u}}^{h}\right\|_{\infty}^{2} & \leq c\left(\left\|\widehat{\mathbf{u}}^{h}-\mathrm{I}^{h} \mathbf{u}\right\|_{\infty}^{2}+\left\|\mathrm{I}^{h} \mathbf{u}\right\|_{\infty}^{2}\right) \leq c\left(h^{-2}\left\|\widehat{\mathbf{u}}^{h}-\mathrm{I}^{h} \mathbf{u}\right\|^{2}+\left\|\mathrm{I}^{h} \mathbf{u}\right\|_{\infty}^{2}\right) \\
& \leq c h^{-2}\left(\left\|\widehat{\mathbf{u}}^{h}-\mathbf{u}\right\|^{2}+\left\|\mathbf{u}-\mathrm{I}^{h} \mathbf{u}\right\|^{2}\right)+c\left\|\mathbf{u}-\mathrm{I}^{h} \mathbf{u}\right\|_{\infty}^{2}+\|\mathbf{u}\|_{\infty}^{2} \leq c\|\mathbf{u}\|_{2}^{2} .
\end{aligned}
$$

Also, we use the triangle inequality, (3.11), (3.10), (3.5), and (3.9) to obtain

$$
\begin{aligned}
\left\|\nabla \widehat{\mathbf{u}}^{h}\right\|_{\infty} & \leq\left\|\nabla \widehat{\mathbf{u}}^{h}-\nabla \mathrm{I}^{h} \mathbf{u}\right\|_{\infty}+\left\|\nabla \mathrm{I}^{h} \mathbf{u}-\nabla \mathbf{u}\right\|_{\infty}+\|\nabla \mathbf{u}\|_{\infty} \\
& \leq c h^{-1}\left(\left\|\nabla \widehat{\mathbf{u}}^{h}-\nabla \mathbf{u}\right\|+\left\|\nabla \mathbf{u}-\nabla \mathrm{I}^{h} \mathbf{u}\right\|\right)+c\|\mathbf{u}\|_{2}+\|\nabla \mathbf{u}\|_{\infty} \\
& \leq c\|\mathbf{u}\|_{2}+\|\nabla \mathbf{u}\|_{\infty} .
\end{aligned}
$$

Therefore, we have

$$
\left\|\widehat{\mathbf{u}}^{h}\left(\boldsymbol{\phi}_{i}\right)-\widehat{\mathbf{u}}^{h}\left(\widehat{\boldsymbol{\phi}}_{i}^{h}\right)\right\|^{2} \leq c\left(\|\mathbf{u}\|_{2}^{2}+\|\nabla \mathbf{u}\|_{\infty}^{2}\right)\left\|\boldsymbol{\phi}_{i}-\widehat{\boldsymbol{\phi}}_{i}^{h}\right\|^{2} .
$$

By combining (3.13) with (3.18), we obtain

$$
\left\|\boldsymbol{\phi}_{n+1}-\widehat{\boldsymbol{\phi}}_{n+1}^{h}\right\|^{2} \leq c_{0}^{2} \Delta t^{2} \sum_{i=0}^{n}\left(\left\|\mathbf{u}\left(\boldsymbol{\phi}_{i}\right)-\widehat{\mathbf{u}}^{h}\left(\boldsymbol{\phi}_{i}\right)\right\|^{2}+\left\|\boldsymbol{\phi}_{i}-\widehat{\boldsymbol{\phi}}_{i}^{h}\right\|^{2}\right),
$$

where $c_{0}$ depends on $\|\mathbf{u}\|_{2}$ and $\|\nabla \mathbf{u}\|_{\infty}$. When $n=0$, (3.19) provides

$$
\left\|\phi_{1}-\widehat{\phi}_{1}^{h}\right\|^{2} \leq c_{0}^{2} \Delta t^{2}\left\|\mathbf{u}\left(\phi_{0}\right)-\widehat{\mathbf{u}}^{h}\left(\phi_{0}\right)\right\|^{2} .
$$

For $n=1,(3.19)$ yields

$$
\left\|\phi_{2}-\widehat{\boldsymbol{\phi}}_{2}^{h}\right\|^{2} \leq c_{0}^{2} \Delta t^{2}\left(\sum_{i=0}^{1}\left\|\mathbf{u}\left(\phi_{i}\right)-\widehat{\mathbf{u}}^{h}\left(\phi_{i}\right)\right\|^{2}+c_{0}^{2} \Delta t^{2}\left\|\mathbf{u}\left(\phi_{0}\right)-\widehat{\mathbf{u}}^{h}\left(\phi_{0}\right)\right\|^{2}\right) .
$$


By induction, we obtain

$$
\left\|\boldsymbol{\phi}_{n+1}-\widehat{\boldsymbol{\phi}}_{n+1}^{h}\right\|^{2} \leq c_{0}^{2} \Delta t^{2} \sum_{i=0}^{n}\left(1+c_{0}^{2} \Delta t^{2}\right)^{n-i}\left\|\mathbf{u}\left(\boldsymbol{\phi}_{i}\right)-\widehat{\mathbf{u}}^{h}\left(\boldsymbol{\phi}_{i}\right)\right\|^{2} .
$$

Now, we use a change of variables to calculate $\left\|\mathbf{u}\left(\phi_{i}\right)-\widehat{\mathbf{u}}^{h}\left(\phi_{i}\right)\right\|$. Let $\phi_{i}(\mathbf{x})=\mathbf{y}$, then $\left|\operatorname{det}\left(\nabla \phi_{i}(\mathbf{x})\right)\right| d \mathbf{x}=d \mathbf{y}$ which implies $d \mathbf{x}=\left|\operatorname{det} \nabla \phi_{i}(\mathbf{x})\right|^{-1} d \mathbf{y}$. Recall (3.6) and take gradients of both sides. Then, we have

$$
\begin{aligned}
\left|\operatorname{det} \nabla \boldsymbol{\phi}_{i}\right| & =\left|\operatorname{tr}\left(I+\Delta t\left(\nabla_{\boldsymbol{\phi}} \mathbf{u}\left(\phi_{i-1}\right)\right)^{t}\right)\right| \cdot\left|\operatorname{det} \nabla \phi_{i-1}\right| \\
& \geq|2-\Delta t|\left|\nabla \cdot \mathbf{u} \|_{\infty}\right| \cdot\left|\operatorname{det} \nabla \phi_{i-1}\right| \\
\cdots & \geq\left|2-\Delta t\|\nabla \cdot \mathbf{u}\|_{\infty}\right|^{i} \cdot\left|\operatorname{det} \nabla \phi_{0}\right|=\left|2-\Delta t\|\nabla \cdot \mathbf{u}\|_{\infty}\right|^{i} .
\end{aligned}
$$

Suppose $\Delta t \leq 1 /\|\nabla \cdot \mathbf{u}\|_{\infty}$ so that $\left|2-\Delta t\|\nabla \cdot \mathbf{u}\|_{\infty}\right|^{-i} \leq 1$. Then, the regularity result in Section 3.1.1 implies

$$
\int_{\Omega}\left|\mathbf{u}\left(\phi_{i}(\mathbf{x})\right)-\widehat{\mathbf{u}}^{h}\left(\phi_{i}(\mathbf{x})\right)\right|^{2} d \mathbf{x} \leq c \int_{\Omega(\mathbf{y})}\left|\mathbf{u}(\mathbf{y})-\widehat{\mathbf{u}}^{h}(\mathbf{y})\right|^{2} d \mathbf{y} \leq c h^{4}\|\mathbf{u}\|_{2}^{2}
$$

Now, assume that

$$
\Delta t \leq \min \left\{\frac{1}{\|\nabla \cdot \mathbf{u}\|_{\infty}}, \frac{1}{c_{0}}\right\}
$$

where $c_{0}=c\left(\|\mathbf{u}\|_{2}+\|\nabla \mathbf{u}\|_{\infty}\right)$. We apply (3.21) to (3.20) and use $\Delta t=1 / N$ to obtain

$$
\begin{aligned}
& \left\|\boldsymbol{\phi}_{n+1}-\widehat{\boldsymbol{\phi}}_{n+1}^{h}\right\|^{2} \leq c c_{0}^{2} \Delta t^{2} h^{4}\|\mathbf{u}\|_{2}^{2} \sum_{i=0}^{n}\left(1+c_{0}^{2} \Delta t^{2}\right)^{n-i} \\
& \quad \leq c c_{0}^{2} \Delta t^{2} h^{4}\|\mathbf{u}\|_{2}^{2} \frac{\left(1+c_{0}^{2} \Delta t^{2}\right)^{N}-1}{1+c_{0}^{2} \Delta t^{2}-1}=c h^{4}\|\mathbf{u}\|_{2}^{2}\left\{\left(1+\frac{c_{0}^{2}}{N^{2}}\right)^{N}-1\right\} .
\end{aligned}
$$

Because $\lim _{N \rightarrow \infty}\left(1+\frac{z}{N}\right)^{N}=e^{z}$,

$$
\left(1+\frac{c_{0}^{2}}{N^{2}}\right)^{N}=\left(1+\frac{i c_{0}}{N}\right)^{N}\left(1-\frac{i c_{0}}{N}\right)^{N} \underset{\text { from below }}{\longrightarrow} e^{i c_{0}} \cdot e^{-i c_{0}}=1,
$$

as $N \rightarrow \infty$. Hence, $\left(1+\frac{c_{0}^{2}}{N^{2}}\right)^{N}-1<2$ and therefore we have

$$
\left\|\phi_{n+1}-\widehat{\phi}_{n+1}^{h}\right\| \leq c h^{2}\|\mathbf{u}\|_{2} .
$$

$\square$

3.1.3. Error estimates for (2.6). Let the partition $\left\{t_{n}\right\}_{n=0}^{N}$ of $[0,1]$ be defined as in Section 3.1.2. In this subsection, we consider the approximate solution of (2.6) by employing an explicit backwards-in-time finite difference scheme, i.e., at time $t=t_{n-1}$,

$$
\begin{aligned}
\boldsymbol{\psi}_{N}\left(t_{n-1}, \mathbf{x}\right) & =\boldsymbol{\psi}_{N}\left(t_{n}, \mathbf{x}\right)+\Delta t \nabla_{\boldsymbol{\phi}} \mathbf{u}\left(\boldsymbol{\phi}_{n-1}(\mathbf{x})\right) \boldsymbol{\psi}_{N}\left(t_{n}, \mathbf{x}\right) \\
\boldsymbol{\psi}_{N}(1, \mathbf{x}) & =(\mathbf{T}(\boldsymbol{\phi}(1, \mathbf{x}))-\mathbf{R}(\mathbf{x})) \nabla_{\boldsymbol{\phi}} \mathbf{T}(\boldsymbol{\phi}(1, \mathbf{x}))
\end{aligned}
$$

Again, we have

$$
\left|\boldsymbol{\psi}\left(t_{n-1}, \mathbf{x}\right)-\boldsymbol{\psi}_{N}\left(t_{n-1}, \mathbf{x}\right)\right| \sim O(\Delta t)
$$


We abbreviate $\boldsymbol{\psi}_{N}\left(t_{n}, \mathbf{x}\right)$ to $\boldsymbol{\psi}_{n}(\mathbf{x})$ and $\widehat{\boldsymbol{\psi}}_{N}^{h}\left(t_{n}, \mathbf{x}\right)$ to $\widehat{\boldsymbol{\psi}}_{n}^{h}(\mathbf{x})$.

Apply the approximations $\widehat{\mathbf{u}}^{h}$ and $\widehat{\phi}^{h}$ from Sections 3.1.1 and 3.1.2 to define the approximation of $\boldsymbol{\psi}$ at time $t=t_{n-1}$ :

$$
\begin{aligned}
& \widehat{\boldsymbol{\psi}}_{n-1}^{h}(\mathbf{x})=\widehat{\boldsymbol{\psi}}_{n}^{h}(\mathbf{x})+\Delta t \nabla_{\boldsymbol{\phi}}^{h} \widehat{\mathbf{u}}^{h}\left(\widehat{\boldsymbol{\phi}}_{n-1}^{h}(\mathbf{x})\right) \widehat{\boldsymbol{\psi}}_{n}^{h}(\mathbf{x}), \\
& \widehat{\boldsymbol{\psi}}^{h}(1, \mathbf{x})=\left(\mathrm{I}^{h} \mathbf{T}\left(\widehat{\boldsymbol{\phi}}^{h}(1, \mathbf{x})\right)-\mathrm{I}^{h} \mathbf{R}(\mathbf{x})\right) \nabla_{\phi} \mathrm{I}^{h} \mathbf{T}\left(\widehat{\boldsymbol{\phi}}^{h}(1, \mathbf{x})\right),
\end{aligned}
$$

where $\nabla^{h}$ is the discrete gradient since $\widehat{\mathbf{u}}^{h}$ is only continuous and a piecewise polynomial on each finite element. Because of (3.23), we need pay attention only to $\boldsymbol{\psi}_{n-1}-\widehat{\boldsymbol{\psi}}_{n-1}^{h}$. Because $\left\|\boldsymbol{\psi}_{n-1}-\widehat{\boldsymbol{\psi}}_{n-1}^{h}\right\|^{2}=\sum_{\tau \in \mathcal{T}_{h}}\left\|\boldsymbol{\psi}_{n-1}-\widehat{\boldsymbol{\psi}}_{n-1}^{h}\right\|_{L^{2}(\tau)}^{2}$, we replace the discrete gradient $\nabla^{h}$ with the exact gradient $\nabla$ on each element.

Now we prove the $L^{2}$-error bound for $\boldsymbol{\psi}_{n-1}-\widehat{\boldsymbol{\psi}}_{n-1}^{h}$.

Proposition 3.5. Suppose that $\Delta t$ is sufficiently small. Then, for each fixed time $t=t_{n}, n=0, \ldots, N$, we have

$$
\left\|\boldsymbol{\psi}_{n}-\widehat{\boldsymbol{\psi}}_{n}^{h}\right\| \leq c h
$$

where $c$ depends of $\|\mathbf{T}\|_{1, \infty}, h\|\mathbf{T}\|_{2},\|\mathbf{R}\|_{\infty}, h\|\mathbf{R}\|_{2},\|\mathbf{u}\|_{2}$, and $\|\nabla \mathbf{u}\|_{\infty}$.

Proof. By (3.22), (3.24), and the Hölder inequality, at time $t=t_{n-1}$ we have

$$
\begin{gathered}
\left\|\boldsymbol{\psi}_{n-1}-\widehat{\boldsymbol{\psi}}_{n-1}^{h}\right\| \\
\leq\left\|\boldsymbol{\psi}_{n}-\widehat{\boldsymbol{\psi}}_{n}^{h}\right\|+\Delta t\left(\sum_{\tau \in \mathcal{T}_{h}}\left\|\nabla_{\phi} \mathbf{u}\left(\boldsymbol{\phi}_{n-1}\right) \boldsymbol{\psi}_{n}-\nabla_{\phi} \widehat{\mathbf{u}}^{h}\left(\widehat{\boldsymbol{\phi}}_{n-1}^{h}\right) \widehat{\boldsymbol{\psi}}_{n}^{h}\right\|_{L^{2}(\tau)}^{2}\right)^{\frac{1}{2}} .
\end{gathered}
$$

First, we consider

$$
\begin{aligned}
& \nabla_{\boldsymbol{\phi}} \mathbf{u}\left(\boldsymbol{\phi}_{n-1}\right) \boldsymbol{\psi}_{n}-\nabla_{\boldsymbol{\phi}} \widehat{\mathbf{u}}^{h}\left(\widehat{\boldsymbol{\phi}}_{n-1}^{h}\right) \widehat{\boldsymbol{\psi}}_{n}^{h}=\underbrace{\nabla_{\phi} \mathbf{u}\left(\boldsymbol{\phi}_{n-1}\right) \boldsymbol{\psi}_{n}-\nabla_{\phi} \widehat{\mathbf{u}}^{h}\left(\boldsymbol{\phi}_{n-1}\right) \boldsymbol{\psi}_{n}}_{(\mathrm{i})} \\
& +\underbrace{\nabla_{\boldsymbol{\phi}} \widehat{\mathbf{u}}^{h}\left(\boldsymbol{\phi}_{n-1}\right) \boldsymbol{\psi}_{n}-\nabla_{\boldsymbol{\phi}} \widehat{\mathbf{u}}^{h}\left(\widehat{\boldsymbol{\phi}}_{n-1}^{h}\right) \boldsymbol{\psi}_{n}}_{(\mathrm{iii})}+\underbrace{\nabla_{n}}_{\underbrace{}_{\boldsymbol{\phi}} \widehat{\mathbf{u}}^{h}\left(\widehat{\boldsymbol{\phi}}_{n-1}^{h}\right) \boldsymbol{\psi}_{n}-\nabla_{\phi} \widehat{\mathbf{u}}^{h}\left(\widehat{\boldsymbol{\phi}}_{n-1}^{h}\right) \widehat{\boldsymbol{\psi}}_{n}^{h}}
\end{aligned}
$$

term by term:

(i) Since $\mathbf{T}(\phi(1, \mathbf{x}))-\mathbf{R}(\mathbf{x})$ and $\nabla_{\boldsymbol{\phi}} \mathbf{T}(\boldsymbol{\phi}(1, \mathbf{x}))$ are bounded, $\boldsymbol{\psi}_{N}(\mathbf{x})=\boldsymbol{\psi}(1, \mathbf{x})$ is bounded. Then, (3.22) and induction imply

$$
\left\|\boldsymbol{\psi}_{n}\right\|_{\infty} \leq\left(1+2 \Delta t\|\nabla \mathbf{u}\|_{\infty}\right)^{N-n}\left\|\boldsymbol{\psi}_{N}\right\|_{\infty} .
$$

Thus, we have

$\left\|\nabla_{\phi} \mathbf{u}\left(\phi_{n-1}\right) \boldsymbol{\psi}_{n}-\nabla_{\phi} \widehat{\mathbf{u}}^{h}\left(\phi_{n-1}\right) \boldsymbol{\psi}_{n}\right\|_{L^{2}(\tau)}^{2} \leq c\left\|\nabla_{\phi} \mathbf{u}\left(\phi_{n-1}\right)-\nabla_{\phi} \widehat{\mathbf{u}}^{h}\left(\phi_{n-1}\right)\right\|_{L^{2}(\tau)}^{2}$, where $c$ is composed of $\left\|\psi_{N}\right\|_{\infty} e^{M_{u}}$ with $M_{u}$ the constant from (3.1), under the assumption $\Delta t \leq 1 /\left(2\|\nabla \mathbf{u}\|_{\infty}\right)$.

(ii) Because $\widehat{\mathbf{u}}^{h}$ is piecewise linear (quadratic) on each element,

$$
\left\|\nabla_{\boldsymbol{\phi}} \widehat{\mathbf{u}}^{h}\left(\phi_{n-1}\right)-\nabla_{\phi} \widehat{\mathbf{u}}^{h}\left(\widehat{\boldsymbol{\phi}}_{n-1}^{h}\right)\right\| \leq c\left\|\nabla \widehat{\mathbf{u}}^{h}\right\|_{\infty}\left\|\phi_{n-1}-\widehat{\boldsymbol{\phi}}_{n-1}^{h}\right\| .
$$

Then, the boundednesses of $\boldsymbol{\psi}_{n}$ from (i) and (3.17) lead us to

$$
\int_{\tau}\left|\nabla_{\phi} \widehat{\mathbf{u}}^{h}\left(\phi_{n-1}\right) \boldsymbol{\psi}_{n}-\nabla_{\boldsymbol{\phi}} \widehat{\mathbf{u}}^{h}\left(\widehat{\boldsymbol{\phi}}_{n-1}^{h}\right) \boldsymbol{\psi}_{n}\right|^{2} d \mathbf{x} \leq c_{0}^{2}\left\|\boldsymbol{\phi}_{n-1}-\widehat{\boldsymbol{\phi}}_{n-1}^{h}\right\|_{L^{2}(\tau)}^{2},
$$

where $c_{0}=c\left(\|\mathbf{u}\|_{2}+\|\nabla \mathbf{u}\|_{\infty}\right)$. 
(iii) By the bound on $\left|\nabla_{\phi} \widehat{\mathbf{u}}^{h}(\phi)\right|$ showed in (ii), we have

$$
\int_{\tau}\left|\nabla_{\boldsymbol{\phi}} \widehat{\mathbf{u}}^{h}\left(\widehat{\boldsymbol{\phi}}_{n-1}^{h}\right) \boldsymbol{\psi}_{n}-\nabla_{\boldsymbol{\phi}} \widehat{\mathbf{u}}^{h}\left(\widehat{\boldsymbol{\phi}}_{n-1}^{h}\right) \widehat{\boldsymbol{\psi}}_{n}^{h}\right|^{2} d \mathbf{x} \leq c_{0}^{2}\left\|\boldsymbol{\psi}_{n}-\widehat{\boldsymbol{\psi}}_{n}^{h}\right\|_{L^{2}(\tau)}^{2},
$$

where $c_{0}=c\left(\|\mathbf{u}\|_{2}+\|\nabla \mathbf{u}\|_{\infty}\right)$.

Gathering the results from (i), (ii), and (iii) yields

$$
\begin{gathered}
\left\|\nabla_{\boldsymbol{\phi}} \mathbf{u}\left(\boldsymbol{\phi}_{n-1}\right) \boldsymbol{\psi}_{n}-\nabla_{\boldsymbol{\phi}} \widehat{\mathbf{u}}^{h}\left(\widehat{\boldsymbol{\phi}}_{n-1}^{h}\right) \widehat{\boldsymbol{\psi}}_{n}^{h}\right\|_{L^{2}(\tau)}^{2} \leq c\left\|\nabla_{\boldsymbol{\phi}} \mathbf{u}\left(\boldsymbol{\phi}_{n-1}\right)-\nabla_{\boldsymbol{\phi}} \widehat{\mathbf{u}}^{h}\left(\boldsymbol{\phi}_{n-1}\right)\right\|_{L^{2}(\tau)}^{2} \\
+c_{0}^{2}\left\|\boldsymbol{\phi}_{n-1}-\widehat{\boldsymbol{\phi}}_{n-1}^{h}\right\|_{L^{2}(\tau)}^{2}+c_{0}^{2}\left\|\boldsymbol{\psi}_{n}-\widehat{\boldsymbol{\psi}}_{n}^{h}\right\|_{L^{2}(\tau)}^{2} .
\end{gathered}
$$

We apply the same change of variables used in the proof of Proposition 3.4 to obtain

$$
\begin{aligned}
& \sum_{\tau \in \mathcal{T}_{h}}\left\|\nabla_{\phi} \mathbf{u}\left(\phi_{n-1}\right)-\nabla_{\phi} \widehat{\mathbf{u}}^{h}\left(\phi_{n-1}\right)\right\|_{L^{2}(\tau)}^{2} \\
& \leq c \sum_{\tau \in \mathcal{T}_{h}} \int_{\tau(\mathbf{y})}\left|\nabla \mathbf{u}(\mathbf{y})-\nabla \widehat{\mathbf{u}}^{h}(\mathbf{y})\right|^{2} d \mathbf{y} \leq c\left\|\nabla \mathbf{u}-\nabla \widehat{\mathbf{u}}^{h}\right\|^{2} \leq c h^{2}\|\mathbf{u}\|_{2}^{2}
\end{aligned}
$$

and apply (3.12) to obtain

$$
\sum_{\tau \in \mathcal{T}_{h}}\left\|\boldsymbol{\phi}_{n-1}-\widehat{\boldsymbol{\phi}}_{n-1}^{h}\right\|_{L^{2}(\tau)}^{2}=\left\|\boldsymbol{\phi}_{n-1}-\widehat{\boldsymbol{\phi}}_{n-1}^{h}\right\|_{L^{2}(\Omega)}^{2} \leq c h^{4}\|\mathbf{u}\|_{2}^{2}
$$

Thus, by (3.27)-(3.29), the inequality in (3.25) becomes

$$
\left\|\boldsymbol{\psi}_{n-1}-\widehat{\boldsymbol{\psi}}_{n-1}^{h}\right\| \leq\left(1+c_{0} \Delta t\right)\left\|\boldsymbol{\psi}_{n}-\widehat{\boldsymbol{\psi}}_{n}^{h}\right\|+c_{1} h \Delta t\|\mathbf{u}\|_{2}
$$

In order to determine the bound for $\left\|\boldsymbol{\psi}_{n}-\widehat{\boldsymbol{\psi}}_{n}^{h}\right\|$, the bound for $\left\|\boldsymbol{\psi}(1, \mathbf{x})-\widehat{\boldsymbol{\psi}}^{h}(1, \mathbf{x})\right\|$ needs to be considered first. From the definition of $\widehat{\boldsymbol{\psi}}^{h}(1, \mathbf{x})$, we have

$$
\boldsymbol{\psi}(1, \mathbf{x})-\widehat{\boldsymbol{\psi}}^{h}(1, \mathbf{x})=\left(\mathbf{T}\left(\boldsymbol{\phi}_{N}\right)-\mathbf{R}\right) \cdot \nabla_{\boldsymbol{\phi}} \mathbf{T}\left(\boldsymbol{\phi}_{N}\right)-\left(\mathrm{I}^{h} \mathbf{T}\left(\widehat{\boldsymbol{\phi}}_{N}^{h}\right)-\mathrm{I}^{h} \mathbf{R}\right) \cdot \nabla_{\boldsymbol{\phi}} \mathrm{I}^{h} \mathbf{T}\left(\widehat{\boldsymbol{\phi}}_{N}^{h}\right) .
$$

Then, on each element $\tau$,

$$
\begin{aligned}
\int_{\tau} \mid & \psi(1, \mathbf{x})-\left.\widehat{\psi}^{h}(1, \mathbf{x})\right|^{2} d \mathbf{x} \\
& \leq c \int_{\tau}\left(\left|\mathbf{T}(\boldsymbol{\phi}(1, \mathbf{x}))-\mathbf{R}-\left(\mathrm{I}^{h} \mathbf{T}(\boldsymbol{\phi}(1, \mathbf{x}))-\mathrm{I}^{h} \mathbf{R}\right)\right|^{2}\right)\left|\nabla_{\boldsymbol{\phi}} \mathbf{T}(\boldsymbol{\phi}(1, \mathbf{x}))\right|^{2} d \mathbf{x} \\
& +c \int_{\tau}\left|\mathrm{I}^{h} \mathbf{T}(\boldsymbol{\phi}(1, \mathbf{x}))-\mathrm{I}^{h} \mathbf{R}-\left(\mathrm{I}^{h} \mathbf{T}\left(\widehat{\phi}^{h}(1, \mathbf{x})\right)-\mathrm{I}^{h} \mathbf{R}\right)\right|^{2}\left|\nabla_{\boldsymbol{\phi}} \mathbf{T}(\boldsymbol{\phi}(1, \mathbf{x}))\right|^{2} d \mathbf{x} \\
& +c \int_{\tau}\left|\mathrm{I}^{h} \mathbf{T}\left(\widehat{\boldsymbol{\phi}}^{h}(1, \mathbf{x})\right)-\mathrm{I}^{h} \mathbf{R}\right|^{2}\left|\nabla_{\boldsymbol{\phi}} \mathbf{T}(\boldsymbol{\phi}(1, \mathbf{x}))-\nabla_{\boldsymbol{\phi}} \mathrm{I}^{h} \mathbf{T}(\boldsymbol{\phi}(1, \mathbf{x}))\right|^{2} d \mathbf{x} \\
& +c \int_{\tau}\left|\mathrm{I}^{h} \mathbf{T}\left(\widehat{\boldsymbol{\phi}}^{h}(1, \mathbf{x})\right)-\mathrm{I}^{h} \mathbf{R}\right|^{2}\left|\nabla_{\phi} \mathrm{I}^{h} \mathbf{T}(\boldsymbol{\phi}(1, \mathbf{x}))-\nabla_{\boldsymbol{\phi}} \mathrm{I}^{h} \mathbf{T}\left(\widehat{\boldsymbol{\phi}}^{h}(1, \mathbf{x})\right)\right|^{2} d \mathbf{x} .
\end{aligned}
$$

We consider (3.31)-(3.34) term by term.

(3.31): Because $\nabla \mathbf{T} \in \mathcal{C}^{0}, \nabla_{\phi} \mathbf{T}(\phi(1, \mathbf{x}))$ is bounded and then the triangle inequality implies, with $c=2\|\nabla \mathbf{T}\|_{\infty}^{2}$,

$$
(3.31) \leq c\left(\left\|\mathbf{T}(\phi(1, \mathbf{x}))-\mathrm{I}^{h} \mathbf{T}(\boldsymbol{\phi}(1, \mathbf{x}))\right\|_{L^{2}(\tau)}^{2}+\left\|\mathbf{R}-\mathrm{I}^{h} \mathbf{R}\right\|_{L^{2}(\tau)}^{2}\right) .
$$


(3.32): By the boundedness of $\nabla \mathbf{T}$, the property $\mathrm{I}^{h} \mathbf{T} \in P_{k}(\tau)$ with (3.14) and (3.15), and (3.10), we have, with $c_{2}$ depending on $h\|\mathbf{T}\|_{2}$ and $\|\mathbf{T}\|_{1, \infty}$,

$$
\begin{aligned}
(3.32) & \leq\|\nabla \mathbf{T}\|_{\infty}^{2} \int_{\tau}\left|\mathrm{I}^{h} \mathbf{T}(\phi(1, \mathbf{x}))-\mathrm{I}^{h} \mathbf{T}\left(\widehat{\phi}^{h}(1, \mathbf{x})\right)\right|^{2} d \mathbf{x} \\
& \leq\|\nabla \mathbf{T}\|_{\infty}^{2}\left\|\mathrm{I}^{h} \mathbf{T}\right\|_{1, \infty}^{2} \int_{\tau}\left|\phi(1, \mathbf{x})-\widehat{\phi}^{h}(1, \mathbf{x})\right|^{2} d \mathbf{x} \\
& \leq c_{2} \int_{\tau}\left|\phi(1, \mathbf{x})-\widehat{\phi}^{h}(1, \mathbf{x})\right|^{2} d \mathbf{x} .
\end{aligned}
$$

(3.33): Similarly to the above, the property of $\mathrm{I}^{h} \mathbf{T}$ being a piecewise polynomial on each $\tau$, the definition of $\widehat{\phi}^{h}\left(t_{k}, \mathbf{x}\right)$, and (3.10) result in

$$
\begin{gathered}
\int_{\tau}\left|\mathrm{I}^{h} \mathbf{T}\left(\widehat{\phi}^{h}(1, \mathbf{x})\right)-\mathrm{I}^{h} \mathbf{R}\right|^{2} d \mathbf{x} \leq 2|\Omega|\left(\left\|\mathrm{I}^{h} \mathbf{T}\right\|_{\infty}^{2}+\left\|\mathrm{I}^{h} \mathbf{R}\right\|_{\infty}^{2}\right) \\
\leq c\left(\|\mathbf{T}\|_{\infty}^{2}+h^{2}\|\mathbf{T}\|_{2}^{2}+\|\mathbf{R}\|_{\infty}^{2}+h^{2}\|\mathbf{R}\|_{2}^{2}\right) .
\end{gathered}
$$

(3.34): By the inverse inequality (3.11) and (3.10), the following holds:

$$
\left\|\nabla \mathrm{I}^{h} \mathbf{T}\right\|_{\infty} \leq c h^{-1}\left\|\mathrm{I}^{h} \mathbf{T}\right\|_{\infty} \leq c h^{-1}\left(\|\mathbf{T}\|_{\infty}+h\|\mathbf{T}\|_{2}\right) .
$$

Because $\mathrm{I}^{h} \mathbf{T}$ is piecewise linear or piecewise quadratic on each $\tau,(3.37)$ and (3.38) result in

$$
(3.34) \leq c h^{-2}\left(\|\mathbf{T}\|_{\infty}^{2}+h^{2}\|\mathbf{T}\|_{2}^{2}\right) \int_{\tau}\left|\phi(1, \mathbf{x})-\widehat{\phi}^{h}(1, \mathbf{x})\right|^{2} d \mathbf{x}
$$

From (3.35)-(3.37) and (3.39), we conclude that

$$
\begin{aligned}
\left\|\boldsymbol{\psi}(1, \mathbf{x})-\widehat{\boldsymbol{\psi}}^{h}(1, \mathbf{x})\right\|^{2} \leq c( & \left\|\mathbf{T}(\boldsymbol{\phi}(1, \mathbf{x}))-\mathrm{I}^{h} \mathbf{T}(\boldsymbol{\phi}(1, \mathbf{x}))\right\|_{1}^{2} \\
& \left.+\left\|\mathbf{R}-\mathrm{I}^{h} \mathbf{R}\right\|^{2}+h^{-2}\left\|\boldsymbol{\phi}(1, \mathbf{x})-\widehat{\boldsymbol{\phi}}^{h}(1, \mathbf{x})\right\|^{2}\right) .
\end{aligned}
$$

Recall the change of variables so that (3.9) and Proposition 3.4 yield

$$
\left\|\boldsymbol{\psi}(1, \mathbf{x})-\widehat{\boldsymbol{\psi}}^{h}(1, \mathbf{x})\right\|^{2} \leq c h^{2}\left(\|\mathbf{T}\|_{2}^{2}+\|\mathbf{R}\|_{2}^{2}+\|\mathbf{u}\|_{2}^{2}\right) .
$$

Let $C_{T R u} \equiv\|\mathbf{T}\|_{2}+\|\mathbf{R}\|_{2}+\|\mathbf{u}\|_{2}$ and assume

$$
\Delta t \leq \min \left\{\frac{1}{2\|\nabla \mathbf{u}\|_{\infty}}, \frac{1}{c_{0}}\right\}
$$

where $c_{0}=c\left(\|\mathbf{u}\|_{2}+\|\nabla \mathbf{u}\|_{\infty}\right)$. Now, we apply the result in (3.40) to (3.30): by induction, we obtain

$$
\begin{aligned}
& \left\|\boldsymbol{\psi}_{n-1}-\widehat{\boldsymbol{\psi}}_{n-1}^{h}\right\| \\
& \quad \leq c h\left(1+c_{0} \Delta t\right)^{N-n+1} C_{T R u}+c_{1} h \Delta t\|\mathbf{u}\|_{2} \sum_{j=0}^{N-n}\left(1+c_{0} \Delta t\right)^{j} \\
& \quad \leq c h\left(1+c_{0} \Delta t\right)^{N-n+1} C_{T R u}+c_{1} h \Delta t\|\mathbf{u}\|_{2} \frac{\left(1+c_{0} \Delta t\right)^{N-n+1}-1}{1+c_{0} \Delta t-1} \\
& \quad \leq h\left(c+c_{1} / c_{0}\right) e^{c_{0} \Delta t(N-n+1)} C_{T R u} \leq h e^{c_{0}}\left(c+c_{1} / c_{0}\right) C_{T R u} .
\end{aligned}
$$


Remark 1. The assumptions on the time step $\Delta t$ given in Propositions 3.4 and 3.5 are consistent. We can summarize both assumptions into

$$
\Delta t \leq \min \left\{\frac{1}{2\|\nabla \mathbf{u}\|_{\infty}}, \frac{1}{c\left(\|\mathbf{u}\|_{2}+\|\nabla \mathbf{u}\|_{\infty}\right)}\right\},
$$

where $c$ does not depend on $\mathbf{u}$ nor any other optimal solution variables or Lagrange multipliers.

3.1.4. Error estimates for (2.7). First, we take a closer look at (2.7) which we express as

$$
\begin{cases}\nabla^{\perp} \eta-\nabla \xi=\mathbf{F}, & \text { in } \Omega \\ \eta=0, & \text { on } \Gamma,\end{cases}
$$

where $\mathbf{F}=\int_{I}\left|\nabla \phi^{-1}(t, \mathbf{x})\right| \boldsymbol{\psi}\left(t, \boldsymbol{\phi}^{-1}(t, \mathbf{x})\right) d t$. In [12], we showed that $\mathbf{F} \in L^{2}(\Omega)$. For given $\mathbf{F} \in L^{2}(\Omega)$, it is easy to see that there exists a unique $(\eta, \xi) \in H_{0}^{1}(\Omega) \times H^{1}(\Omega) / \mathbb{R}$ satisfying (3.41). We apply the FOSLL* method introduced in [5]. The basic idea of FOSLL* can be explained by considering the dual of a linear system of equations $A \mathbf{x}=\mathbf{b}$. Least-squares methods minimize $\|A \mathbf{x}-\mathbf{b}\|^{2}$ and the dual of this method involves the system $A A^{t} \mathbf{y}=\mathbf{b}$ with $\mathbf{x}=A^{t} \mathbf{y}$ and the minimization of the functional $\left\langle A^{t} \mathbf{y}, A^{t} \mathbf{y}\right\rangle-2\langle\mathbf{b}, \mathbf{y}\rangle$ which is equivalent to minimizing $\left\|A^{t} \mathbf{y}-\mathbf{x}\right\|^{2}$.

For a given first-order system $L \mathbf{u}=\mathbf{f}$, the least-squares method requires $H^{1}$-norm equivalence of $\|L \mathbf{u}\|^{2}$. In (3.41), we can easily see that $\left\|\nabla^{\perp} \eta-\nabla \xi\right\|^{2} \sim\|\eta\|_{1}^{2}+\|\xi\|_{1}^{2}$. So the least-squares approximation exists. However, we do not obtain any convergence of the approximate solution in finite-dimensional subspaces by minimizing $\| \mathcal{L} \mathbf{u}-$ $\mathbf{f} \|$. Therefore, we use FOSLL* for (3.41) because the FOSLL* approach minimizes $\left\|L^{*} \mathbf{w}-\mathbf{u}\right\|^{2}$ which provides an $L^{2}$-approximation of $\mathbf{u}$ and an $L^{2}$-error estimate for the approximation.

We write (3.41) as

$$
\mathcal{L}(\eta, \xi)^{t}=\left[\begin{array}{ll}
\nabla^{\perp} & -\nabla
\end{array}\right]\left[\begin{array}{c}
\eta \\
\xi
\end{array}\right]=\mathbf{F}
$$

and define the domain of $\mathcal{L}$ as $D(\mathcal{L})=H_{0}^{1}(\Omega) \times H^{1}(\Omega) / \mathbb{R}$. Now, we consideer the corresponding dual problem

$$
\mathcal{L}^{*} \mathbf{w}=\left[\begin{array}{c}
\nabla \times \\
\nabla \cdot
\end{array}\right] \mathbf{w}=\left[\begin{array}{l}
\eta \\
\xi
\end{array}\right]
$$

with the domain of $\mathcal{L}^{*}, D\left(\mathcal{L}^{*}\right)=\{\mathbf{w} \in H(\nabla \times) \cap H(\nabla \cdot): \mathbf{n} \cdot \mathbf{w}=0$ on $\Gamma\}$. Lemma 2.1 in [5] yields that there is a unique $\mathbf{w} \in D\left(\mathcal{L}^{*}\right)$ satisfying (3.43).

By $[9$, Proposition 3.1$]$, we have $\left\|\mathcal{L}^{*} \mathbf{w}\right\| \sim\|\mathbf{w}\|_{1}$ which guarantees the existence of a least-squares approximation. The FOSLL* method minimizes the least-squares functional $\left\|\mathcal{L}^{*} \mathbf{w}-(\eta, \xi)^{t}\right\|^{2}$ in the weak sense, that is, we look for the solution of the corresponding variational formulation as follows: find $\mathbf{w} \in D\left(\mathcal{L}^{*}\right)$ such that

$$
\left\langle\mathcal{L}^{*} \mathbf{w}, \mathcal{L}^{*} \mathbf{v}\right\rangle=\left\langle(\eta, \xi)^{t}, \mathcal{L}^{*} \mathbf{v}\right\rangle=\left\langle\mathcal{L}(\eta, \xi)^{t}, \mathbf{v}\right\rangle=\langle\mathbf{F}, \mathbf{v}\rangle \quad \forall \mathbf{v} \in D\left(\mathcal{L}^{*}\right)
$$

This equation shows that we only need the original right-hand side $\mathbf{F}$ to solve the variational formulation of the least-squares problem. 
Now, we substitute (3.43) into (3.42) to obtain

$$
\mathcal{L}\left[\begin{array}{l}
\eta \\
\xi
\end{array}\right]=\mathcal{L L}^{*} \mathbf{w}=\left[\begin{array}{ll}
\nabla^{\perp} & -\nabla
\end{array}\right]\left[\begin{array}{c}
\nabla \times \\
\nabla \cdot
\end{array}\right] \mathbf{w}=-\Delta \mathbf{w}=\mathbf{F} .
$$

For a given $\mathbf{F} \in L^{2}(\Omega), \mathbf{w}$ in fact belongs to $\left[H^{2}(\Omega)\right]^{2}$.

Define $\mathcal{V}^{h}=\left\{\mathbf{w} \in \mathcal{C}^{0}(\Omega):\left.\mathbf{w}\right|_{\tau} \in P_{k}(\tau), k \geq 1\right\} \subset H_{N}^{1}(\Omega)$ and let

$$
\widehat{\mathbf{w}}^{h}=\arg \min _{\widetilde{\mathbf{w}}^{h} \in \mathcal{V}^{h}}\left\|\mathcal{L}^{*} \widetilde{\mathbf{w}}^{h}-(\eta, \xi)^{t}\right\| .
$$

Then, we have an $L^{2}$-error estimate for $(\eta, \xi)$ in the next theorem.

Proposition 3.6. Let $\mathbf{w}$ and $\widehat{\mathbf{w}}^{h}$ satisfy (3.45) and (3.46), respectively. Let $\left(\widehat{\eta}^{h}, \widehat{\xi}^{h}\right)^{t}=\mathcal{L}^{*} \widehat{\mathbf{w}}^{h}$. Then, we have

$$
\left\|(\eta, \xi)-\left(\widehat{\eta}^{h}, \widehat{\xi}^{h}\right)\right\| \leq c h\|\mathbf{w}\|_{2} .
$$

Proof. Using the $H^{1}$-norm equivalence of the $\mathcal{L}^{*}$-operator and standard regularity properties, we have

$$
\left\|(\eta, \xi)-\left(\widehat{\eta}^{h}, \widehat{\xi}^{h}\right)\right\|=\left\|\mathcal{L}^{*} \mathbf{w}-\mathcal{L}^{*} \widehat{\mathbf{w}}^{h}\right\| \leq c h\|\mathbf{w}\|_{2} .
$$

$\square$

3.1.5. Error estimates for (2.8). The system (2.8) can be rewritten as the equivalent first-order systems

$$
\left\{\begin{aligned}
\mathbf{p}-\nabla f & =\mathbf{0} & & \text { in } \Omega \\
-\frac{1}{\beta_{f}} \nabla \cdot \mathbf{p}+\beta_{f} f & =\frac{1}{\alpha_{f_{1}} \beta_{f}}(\sigma-\xi) & & \text { in } \Omega \\
\frac{1}{\beta_{f}} \nabla \times \mathbf{p} & =0 & & \text { in } \Omega \\
\mathbf{n} \cdot \mathbf{p} & =0 & & \text { on } \Gamma
\end{aligned}\right.
$$

and

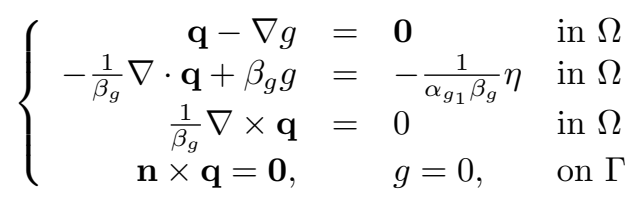

by introducing flux variables, $\mathbf{p}, \mathbf{q}$, and scalings $\beta_{f}=\sqrt{\alpha_{f_{0}} / \alpha_{f_{1}}}$ and $\beta_{g}=\sqrt{\alpha_{g_{0}} / \alpha_{g_{1}}}$. The third equations in (3.47) and (3.48), i.e., $\nabla \times \mathbf{p}=\mathbf{0}$ and $\nabla \times \mathbf{q}=\mathbf{0}$, respectively, are auxiliary equations introduced to improve the regularity of $\mathbf{p}$ and $\mathbf{q}$, respectively. The least-squares method minimizes the residual functionals

$$
\mathcal{P}(f, \mathbf{p} ; \sigma, \xi)=\|\mathbf{p}-\nabla f\|^{2}+\left\|-\frac{1}{\beta_{f}} \nabla \cdot \mathbf{p}+\beta_{f} f-\frac{1}{\alpha_{f_{1}} \beta_{f}}(\sigma-\xi)\right\|^{2}+\frac{1}{\beta_{f}^{2}}\|\nabla \times \mathbf{p}\|^{2}
$$

and

$$
\mathcal{Q}(g, \mathbf{q} ; \eta)=\|\mathbf{q}-\nabla g\|^{2}+\left\|-\frac{1}{\beta_{g}} \nabla \cdot \mathbf{q}+\beta_{g} g+\frac{1}{\alpha_{g_{1}} \beta_{g}} \eta\right\|^{2}+\frac{1}{\beta_{g}^{2}}\|\nabla \times \mathbf{q}\|^{2},
$$

respectively. The corresponding weak formulations are given by: find $(f, \mathbf{p}) \in$ $H^{1}(\Omega) / \mathbb{R} \times H_{N}^{1}(\Omega)$ satisfying

$$
\begin{aligned}
& \langle\mathbf{p}-\nabla f, \mathbf{r}-\nabla r\rangle+\left\langle-\frac{1}{\beta_{f}} \nabla \cdot \mathbf{p}+\beta_{f} f,-\frac{1}{\beta_{f}} \nabla \cdot \mathbf{r}+\beta_{f} r\right\rangle \\
& \quad+\frac{1}{\beta_{f}^{2}}\langle\nabla \times \mathbf{p}, \nabla \times \mathbf{r}\rangle=\left\langle-\frac{1}{\alpha_{f_{1}} \beta_{f}}(\sigma-\xi),-\frac{1}{\beta_{f}} \nabla \cdot \mathbf{r}+\beta_{f} r\right\rangle
\end{aligned}
$$


for all $(r, \mathbf{r}) \in H^{1}(\Omega) / \mathbb{R} \times H_{N}^{1}(\Omega)$, and find $(g, \mathbf{q}) \in H_{0}^{1}(\Omega) \times H_{D}^{1}(\Omega)$ satisfying

$$
\begin{array}{r}
\langle\mathbf{q}-\nabla g, \mathbf{s}-\nabla s\rangle+\left\langle-\frac{1}{\beta_{g}} \nabla \cdot \mathbf{q}+\beta_{g} g,-\frac{1}{\beta_{g}} \nabla \cdot \mathbf{s}+\beta_{g} s\right\rangle \\
+\frac{1}{\beta_{g}^{2}}\langle\nabla \times \mathbf{q}, \nabla \times \mathbf{s}\rangle=\left\langle-\frac{1}{\alpha_{g_{1}} \beta_{g}} \eta,-\frac{1}{\beta_{g}} \nabla \cdot \mathbf{s}+\beta_{g} s\right\rangle
\end{array}
$$

for all $(s, \mathbf{s}) \in H_{0}^{1}(\Omega) \times H_{D}^{1}(\Omega)$, respectively.

Define finite-dimensional subspaces $\mathcal{X}^{h} \times X^{h} \subset H^{1}(\Omega) / \mathbb{R} \times H_{N}^{1}(\Omega)$ and $\mathcal{Y}^{h} \times Y^{h} \subset$ $H_{0}^{1}(\Omega) \times H_{D}^{1}(\Omega)$. Then, we consider the finite-dimensional approximate solutions $\left(\widehat{f}^{h}, \widehat{\mathbf{p}}^{h}\right) \in \mathcal{X}^{h} \times X^{h}$ such that

$$
\begin{array}{r}
\left\langle\widehat{\mathbf{p}}^{h}-\nabla \widehat{f}^{h}, \mathbf{r}^{h}-\nabla r^{h}\right\rangle+\left\langle-\frac{1}{\beta_{f}} \nabla \cdot \widehat{\mathbf{p}}^{h}+\beta_{f} \widehat{f}^{h},-\frac{1}{\beta_{f}} \nabla \cdot \mathbf{r}^{h}+\beta_{f} r^{h}\right\rangle \\
+\frac{1}{\beta_{f}^{2}}\left\langle\nabla \times \widehat{\mathbf{p}}^{h}, \nabla \times \mathbf{r}^{h}\right\rangle=\left\langle-\frac{1}{\alpha_{f_{1} \beta_{f}}}(\sigma-\xi),-\frac{1}{\beta_{f}} \nabla \cdot \mathbf{r}^{h}+\beta_{f} r^{h}\right\rangle
\end{array}
$$

for all $\left(r^{h}, \mathbf{r}^{h}\right) \in \mathcal{X}^{h} \times X^{h}$, and $\left(\widehat{g}^{h}, \widehat{\mathbf{q}}^{h}\right) \in \mathcal{Y}^{h} \times Y^{h}$ such that

$$
\begin{gathered}
\left\langle\widehat{\mathbf{q}}-\nabla \widehat{g}^{h}, \mathbf{s}^{h}-\nabla s^{h}\right\rangle+\left\langle-\frac{1}{\beta_{g}} \nabla \cdot \widehat{\mathbf{q}}^{h}+\beta_{g} \widehat{g}^{h},-\frac{1}{\beta_{g}} \nabla \cdot \mathbf{s}^{h}+\beta_{g} s^{h}\right\rangle \\
+\frac{1}{\beta_{g}^{2}}\left\langle\nabla \times \widehat{\mathbf{q}}^{h}, \nabla \times \mathbf{s}^{h}\right\rangle=\left\langle-\frac{1}{\alpha_{g_{1}} \beta_{g}} \eta,-\frac{1}{\beta_{g}} \nabla \cdot \mathbf{s}^{h}+\beta_{g} s^{h}\right\rangle
\end{gathered}
$$

for all $\left(s^{h}, \mathbf{s}^{h}\right) \in \mathcal{Y}^{h} \times Y^{h}$. The next result is well known.

Proposition 3.7. Let $(f, \mathbf{p}),(g, \mathbf{q}),\left(\widehat{f}^{h}, \widehat{\mathbf{p}}^{h}\right)$, and $\left(\widehat{g}^{h}, \mathbf{q}^{h}\right)$ satisfy (3.49), (3.50), (3.51), and (3.52), respectively. Then, we have

$$
\left\|f-\widehat{f}^{h}\right\|_{1}+\left\|\mathbf{p}-\widehat{\mathbf{p}}^{h}\right\|_{1} \leq c h\|\sigma-\xi\| \quad \text { and } \quad\left\|g-\widehat{g}^{h}\right\|_{1}+\left\|\mathbf{q}-\widehat{\mathbf{q}}^{h}\right\|_{1} \leq c h\|\eta\| .
$$

3.2. Error estimates for the fully-coupled discrete optimal solution. In this section, we use the results of Section 3.1 to treat a fully discretized optimality system. The goal is to study the convergence of the approximate solution.

Let $\left(\mathbf{u}^{h}, \boldsymbol{\phi}_{N}^{h}, \boldsymbol{\psi}_{N}^{h}, \xi^{h}, \eta^{h}, f^{h}, g^{h}\right) \in \mathcal{U}^{h} \times\left(\Phi^{h}\right)^{2} \times \mathcal{V}^{h} \times \mathcal{X}^{h} \times \mathcal{Y}^{h}$ satisfy

$$
\left\langle\nabla \cdot \mathbf{u}^{h}, \nabla \cdot \mathbf{v}^{h}\right\rangle+\left\langle\nabla \times \mathbf{u}^{h}, \nabla \times \mathbf{v}^{h}\right\rangle=\left\langle f^{h}-1, \nabla \cdot \mathbf{v}^{h}\right\rangle+\left\langle g^{h}, \nabla \times \mathbf{v}^{h}\right\rangle
$$

for all $\mathbf{v}^{h} \in \mathcal{U}^{h}$,

$$
\left\{\begin{aligned}
\phi_{N}^{h}\left(t_{n+1}, \mathbf{x}\right) & =\phi_{N}^{h}\left(t_{n}, \mathbf{x}\right)+\Delta t \mathbf{u}^{h}\left(\phi_{N}^{h}\left(t_{n}, \mathbf{x}\right)\right) \\
\phi_{N}^{h}\left(t_{0}, \mathbf{x}\right) & =\mathbf{x}
\end{aligned}\right.
$$

for $n=0, \cdots, N-1$,

$$
\left\{\begin{aligned}
\boldsymbol{\psi}_{N}^{h}\left(t_{n-1}, \mathbf{x}\right) & =\boldsymbol{\psi}_{N}^{h}\left(t_{n}, \mathbf{x}\right)+\Delta t \nabla_{\boldsymbol{\phi}} \mathbf{u}^{h}\left(\boldsymbol{\phi}_{N}^{h}\left(t_{n-1}, \mathbf{x}\right)\right) \boldsymbol{\psi}_{N}^{h}\left(t_{n}, \mathbf{x}\right) \\
\boldsymbol{\psi}_{N}^{h}\left(t_{N}, \mathbf{x}\right) & =\left(\mathrm{I}^{h} \mathbf{T}\left(\boldsymbol{\phi}_{N}^{h}\left(t_{N}, \mathbf{x}\right)\right)-\mathrm{I}^{h} \mathbf{R}(\mathbf{x})\right) \nabla_{\phi} \mathrm{I}^{h} \mathbf{T}\left(\boldsymbol{\phi}_{N}^{h}\left(t_{N}, \mathbf{x}\right)\right)
\end{aligned}\right.
$$

for $n=1, \cdots, N,\left(\eta^{h}, \xi^{h}\right)^{t}=\mathcal{L}^{*} \mathbf{w}^{h}$ such that $\mathbf{w}^{h}$ satisfies

$$
\left\langle\mathcal{L}^{*} \mathbf{w}^{h}, \mathcal{L}^{*} \mathbf{v}^{h}\right\rangle=\left\langle(\underline{\eta}, \underline{\xi})^{t}, \mathcal{L}^{*} \mathbf{v}^{h}\right\rangle=\left\langle\mathbf{F}_{N}^{h}, \mathbf{v}^{h}\right\rangle \quad \forall \mathbf{v}^{h} \in \mathcal{V}^{h},
$$

where $\mathbf{F}_{N}^{h}=\Delta t \sum_{n=0}^{N}\left|\operatorname{det} \nabla \boldsymbol{\phi}_{N}^{-1}\left(t_{k}, \mathbf{x}\right)\right| \boldsymbol{\psi}_{N}^{h}\left(t, \boldsymbol{\phi}_{N}^{-1}\left(t_{n}, \mathbf{x}\right)\right)$ with $\boldsymbol{\phi}_{N}$ from (3.6) and $(\underline{\eta}, \underline{\xi})^{t} \in D(\mathcal{L})$ satisfying the $L^{2}$-decomposition (see [9] for details)

$$
\nabla^{\perp} \underline{\eta}-\nabla \underline{\xi}=\mathbf{F}_{N}^{h}
$$




$$
\begin{gathered}
\left\langle\mathbf{p}^{h}-\nabla f^{h}, \mathbf{r}^{h}-\nabla r^{h}\right\rangle+\left\langle-\frac{1}{\beta_{f}} \nabla \cdot \mathbf{p}^{h}+\beta_{f} f^{h},-\frac{1}{\beta_{f}} \nabla \cdot \mathbf{r}^{h}+\beta_{f} r^{h}\right\rangle \\
+\frac{1}{\beta_{f}^{2}}\left\langle\nabla \times \mathbf{p}^{h}, \nabla \times \mathbf{r}^{h}\right\rangle=\left\langle-\frac{1}{\alpha_{f_{1}} \beta_{f}}\left(\sigma^{h}-\xi^{h}\right),-\frac{1}{\beta_{f}} \nabla \cdot \mathbf{r}^{h}+\beta_{f} r^{h}\right\rangle
\end{gathered}
$$

for all $\left(r^{h}, \mathbf{r}^{h}\right) \in \mathcal{X}^{h} \times X^{h}$, and

$$
\begin{gathered}
\left\langle\mathbf{q}-\nabla g^{h}, \mathbf{s}^{h}-\nabla s^{h}\right\rangle+\left\langle-\frac{1}{\beta_{g}} \nabla \cdot \mathbf{q}^{h}+\beta_{g} g^{h},-\frac{1}{\beta_{g}} \nabla \cdot \mathbf{s}^{h}+\beta_{g} s^{h}\right\rangle \\
+\frac{1}{\beta_{g}^{2}}\left\langle\nabla \times \mathbf{q}^{h}, \nabla \times \mathbf{s}^{h}\right\rangle=\left\langle-\frac{1}{\alpha_{g_{1}} \beta_{g}} \eta^{h},-\frac{1}{\beta_{g}} \nabla \cdot \mathbf{s}^{h}+\beta_{g} s^{h}\right\rangle,
\end{gathered}
$$

for all $\left(s^{h}, \mathbf{s}^{h}\right) \in \mathcal{Y}^{h} \times Y^{h}$, where

$$
\sigma^{h}=\alpha_{f_{0}}+\frac{1}{|\Omega|} \int_{\Omega} \xi^{h} d \mathbf{x} .
$$

Now, we present several results in order to show the convergence of the above discrete approximation $\left(\mathbf{u}^{h}, \boldsymbol{\phi}_{N}^{h}, \boldsymbol{\psi}_{N}^{h}, \xi^{h}, \eta^{h}, f^{h}, g^{h}\right)$ to the optimal solution $(\mathbf{u}, \boldsymbol{\phi}, \boldsymbol{\psi}, \xi$, $\eta, f, g)$ of the optimality system $(2.4)-(2.8)$. have

Proposition 3.8. Let $\mathbf{u}$ and $\mathbf{u}^{h}$ satisfy (3.3) and (3.53), respectively. Then, we

$$
\left\|\mathbf{u}-\mathbf{u}^{h}\right\|_{1} \leq c h\|\mathbf{u}\|_{2}+c\left(\left\|f-f^{h}\right\|+\left\|g-g^{h}\right\|\right) .
$$

Proof. By the triangle inequality and Proposition 3.2, we have

$$
\left\|\mathbf{u}-\mathbf{u}^{h}\right\|_{1} \leq\left\|\mathbf{u}-\widehat{\mathbf{u}}^{h}\right\|_{1}+\left\|\widehat{\mathbf{u}}^{h}-\mathbf{u}^{h}\right\|_{1} \leq c h\|\mathbf{u}\|_{2}+\left\|\widehat{\mathbf{u}}^{h}-\mathbf{u}^{h}\right\|_{1} .
$$

We subtract (3.53) from (3.4) to obtain

$$
\begin{gathered}
\left\langle\nabla \cdot \widehat{\mathbf{u}}^{h}-\nabla \cdot \mathbf{u}^{h}, \nabla \cdot \mathbf{v}^{h}\right\rangle+\left\langle\nabla \times \widehat{\mathbf{u}}^{h}-\nabla \times \mathbf{u}^{h}, \nabla \times \mathbf{v}^{h}\right\rangle \\
=\left\langle f-f^{h}, \nabla \cdot \mathbf{v}^{h}\right\rangle+\left\langle g-g^{h}, \nabla \times \mathbf{v}^{h}\right\rangle .
\end{gathered}
$$

Setting $\mathbf{v}^{h}=\widehat{\mathbf{u}}^{h}-\mathbf{u}^{h}$ yields

$$
c\left|\widehat{\mathbf{u}}^{h}-\mathbf{u}^{h}\right|_{1} \leq\left\|\nabla \cdot\left(\widehat{\mathbf{u}}^{h}-\mathbf{u}^{h}\right)\right\|+\left\|\nabla \times\left(\widehat{\mathbf{u}}^{h}-\mathbf{u}^{h}\right)\right\| \leq\left\|f-f^{h}\right\|+\left\|g-g^{h}\right\|,
$$

where the first inequality holds because $\mathbf{n} \cdot\left(\widehat{\mathbf{u}}^{h}-\mathbf{u}^{h}\right)=0$ on the boundary.

In the next result, we consider $\phi$ and $\phi_{N}^{h}$.

Proposition 3.9. Let $\phi$ and $\phi_{N}^{h}$ satisfy (2.5) and (3.54), respectively. If $\Delta t$ is sufficiently small, then

$$
\left\|\phi-\phi_{N}^{h}\right\| \leq c\left(\Delta t+h^{2}\right)+c_{1} \sqrt{\Delta t}\left\|\widehat{\mathbf{u}}^{h}-\mathbf{u}^{h}\right\|
$$

where $c_{1}$ only depends on $\|\mathbf{u}\|_{2}$ and $\|\nabla \mathbf{u}\|_{\infty}$.

Proof. The triangle inequality, elementary properties of the finite difference scheme, and Proposition 3.4 imply

$$
\begin{aligned}
\left\|\phi-\phi_{N}^{h}\right\| & \leq\left\|\phi-\phi_{N}\right\|+\left\|\phi_{N}-\widehat{\phi}_{N}^{h}\right\|+\left\|\widehat{\phi}_{N}^{h}-\phi_{N}^{h}\right\| \\
& \leq c\left(\Delta t+h^{2}\right)+\left\|\widehat{\phi}_{N}^{h}-\phi_{N}^{h}\right\|,
\end{aligned}
$$

where $\phi_{N}$ and $\widehat{\phi}_{N}^{h}$ are defined as in (3.6) and (3.8), respectively. For simplicity of notation, from now on we abbreviate $\widehat{\boldsymbol{\phi}}_{N}^{h}\left(t_{n}, \mathbf{x}\right)$ and $\boldsymbol{\phi}_{N}^{h}\left(t_{n}, \mathbf{x}\right)$ to $\widehat{\boldsymbol{\phi}}_{n}^{h}(\mathbf{x})$ and $\boldsymbol{\phi}_{n}^{h}(\mathbf{x})$, 
respectively. The overall process of this proof is similar to the proof of Proposition 3.4. By definition, we have

$$
\left\|\widehat{\boldsymbol{\phi}}_{n+1}^{h}-\boldsymbol{\phi}_{n+1}^{h}\right\|^{2} \leq 2 \Delta t^{2} \sum_{i=0}^{n}\left\|\widehat{\mathbf{u}}^{h}\left(\widehat{\boldsymbol{\phi}}_{i}^{h}\right)-\mathbf{u}^{h}\left(\boldsymbol{\phi}_{i}^{h}\right)\right\|^{2}
$$

The triangle inequality and (3.14)-(3.17) yield

$$
\begin{aligned}
\left\|\widehat{\mathbf{u}}^{h}\left(\widehat{\boldsymbol{\phi}}_{i}^{h}\right)-\mathbf{u}^{h}\left(\boldsymbol{\phi}_{i}^{h}\right)\right\|^{2} & \leq 2\left\|\widehat{\mathbf{u}}^{h}\left(\widehat{\boldsymbol{\phi}}_{i}^{h}\right)-\widehat{\mathbf{u}}^{h}\left(\boldsymbol{\phi}_{i}^{h}\right)\right\|^{2}+2\left\|\widehat{\mathbf{u}}^{h}\left(\boldsymbol{\phi}_{i}^{h}\right)-\mathbf{u}^{h}\left(\boldsymbol{\phi}_{i}^{h}\right)\right\|^{2} \\
& \leq 2 c_{0}^{2}\left\|\widehat{\boldsymbol{\phi}}_{i}^{h}-\boldsymbol{\phi}_{i}^{h}\right\|^{2}+2\left\|\widehat{\mathbf{u}}^{h}\left(\boldsymbol{\phi}_{i}^{h}\right)-\mathbf{u}^{h}\left(\boldsymbol{\phi}_{i}^{h}\right)\right\|^{2}
\end{aligned}
$$

where $c_{0}=c\|\mathbf{u}\|_{2}+\|\nabla \mathbf{u}\|_{\infty}$. We use the similar change of variables used in (3.21) and the inverse inequality

$$
\begin{aligned}
\left|\operatorname{det} \nabla \boldsymbol{\phi}_{i}^{h}\right| & =\left|\operatorname{tr}\left(I+\Delta t\left(\nabla_{\boldsymbol{\phi}} \mathbf{u}^{h}\left(\boldsymbol{\phi}_{i-1}^{h}\right)\right)^{t}\right)\right| \cdot\left|\operatorname{det} \nabla \boldsymbol{\phi}_{i-1}^{h}\right| \\
& \geq\left|2-\Delta t\left\|\nabla \cdot \mathbf{u}^{h}\right\|_{\infty}\right| \cdot\left|\operatorname{det} \nabla \boldsymbol{\phi}_{i-1}^{h}\right| \\
\cdots & \geq\left|2-\Delta t\left\|\nabla \cdot \mathbf{u}^{h}\right\|_{\infty}\right|^{i} \cdot\left|\operatorname{det} \nabla \boldsymbol{\phi}_{0}^{h}\right|=\left|2-\Delta t\left\|\nabla \cdot \mathbf{u}^{h}\right\|_{\infty}\right|^{i}
\end{aligned}
$$

to obtain

$$
\left\|\widehat{\mathbf{u}}^{h}\left(\widehat{\boldsymbol{\phi}}_{i}^{h}\right)-\mathbf{u}^{h}\left(\boldsymbol{\phi}_{i}^{h}\right)\right\|^{2} \leq 2 c_{0}^{2}\left\|\widehat{\boldsymbol{\phi}}_{i}^{h}-\boldsymbol{\phi}_{i}^{h}\right\|^{2}+2 \gamma^{i}\left\|\widehat{\mathbf{u}}^{h}-\mathbf{u}^{h}\right\|^{2},
$$

where $\gamma=1 /\left|2-\Delta t\left\|\nabla \cdot \mathbf{u}^{h}\right\|_{\infty}\right|$. Therefore

$$
\left\|\widehat{\boldsymbol{\phi}}_{k+1}^{h}-\boldsymbol{\phi}_{k+1}^{h}\right\|^{2} \leq 4 \Delta t^{2} \sum_{i=0}^{k}\left(c_{0}^{2}\left\|\widehat{\boldsymbol{\phi}}_{i}^{h}-\boldsymbol{\phi}_{i}^{h}\right\|^{2}+\gamma^{i}\left\|\widehat{\mathbf{u}}^{h}-\mathbf{u}^{h}\right\|^{2}\right) .
$$

Assume $\Delta t \leq \min \left\{\frac{1}{2 c_{0}}, \frac{1}{\left\|\nabla \cdot \mathbf{u}^{h}\right\|_{\infty}}, \frac{h}{c_{I}\left\|\nabla \cdot \mathbf{u}^{h}\right\|}\right\}$, where the constant $c_{I}$ is from the inverse inequality $\|\cdot\|_{\infty} \leq c_{I} h^{-1}\|\cdot\|$. Then,

$$
|2-\Delta t|\|\nabla \cdot \mathbf{u}\|_{\infty}|\geq| 2-c_{I} \Delta t h^{-1}\|\nabla \cdot \mathbf{u}\| \mid .
$$

Then $\gamma<1$, induction, and $2 c_{0} \Delta t \leq 1$ yield

$$
\begin{aligned}
& \left\|\widehat{\phi}_{n+1}^{h}-\phi_{n+1}^{h}\right\|^{2} \leq 4 \Delta t^{2} \sum_{i=0}^{n}\left(1+4 c_{0}^{2} \Delta t^{2}\right)^{i}\left\|\widehat{\mathbf{u}}^{h}-\mathbf{u}^{h}\right\|^{2} \\
& \leq 4 \Delta t^{2}\left\|\widehat{\mathbf{u}}^{h}-\mathbf{u}^{h}\right\|^{2} \sum_{i=0}^{n}\left(1+2 c_{0} \Delta t\right)^{i}=2 \Delta t\left\|\widehat{\mathbf{u}}^{h}-\mathbf{u}^{h}\right\|^{2} \frac{\left(1+2 c_{0} \Delta t\right)^{n+1}-1}{c_{0}} .
\end{aligned}
$$

The above results in

$$
\begin{gathered}
\left\|\widehat{\boldsymbol{\phi}}_{N}^{h}-\boldsymbol{\phi}_{N}^{h}\right\|^{2}=\Delta t \sum_{n=0}^{N}\left\|\widehat{\boldsymbol{\phi}}_{N}^{h}\left(t_{n}, \mathbf{x}\right)-\boldsymbol{\phi}_{N}^{h}\left(t_{n}, \mathbf{x}\right)\right\|^{2} \\
\leq 2 c_{0}^{-1} \Delta t^{2}\left\|\widehat{\mathbf{u}}^{h}-\mathbf{u}^{h}\right\|^{2} \sum_{n=0}^{N}\left(\left(1+2 c_{0} \Delta t\right)^{n}-1\right) \leq \Delta t c_{0}^{-2} e^{2 c_{0}}\left\|\widehat{\mathbf{u}}^{h}-\mathbf{u}^{h}\right\|^{2} .
\end{gathered}
$$

Thus, by applying the above to (3.59), the proof is complete. $\square$

Now, we focus on the Lagrange multipliers. 
Proposition 3.10. Let $\boldsymbol{\psi}$ and $\boldsymbol{\psi}_{N}^{h}$ satisfy (2.6) and (3.55), respectively. Assume that $\Delta t$ is sufficiently small. Then, we have

$$
\left\|\boldsymbol{\psi}-\boldsymbol{\psi}_{N}^{h}\right\| \leq c(\Delta t+h)+c_{0} \Delta t\left\|\widehat{\mathbf{u}}^{h}-\mathbf{u}^{h}\right\|_{1},
$$

where $c_{0}$ depends only on $\|\mathbf{u}\|_{2},\|\nabla \mathbf{T}\|_{\infty},\|\mathbf{T}\|_{\infty}$, and $\|\mathbf{R}\|_{\infty}$.

Proof. By the triangle inequality, elementary properties of the finite difference scheme, and Proposition 3.5, we have

$$
\begin{aligned}
\left\|\boldsymbol{\psi}-\boldsymbol{\psi}_{N}^{h}\right\| & \leq\left\|\boldsymbol{\psi}-\boldsymbol{\psi}_{N}\right\|+\left\|\boldsymbol{\psi}_{N}-\widehat{\boldsymbol{\psi}}_{N}^{h}\right\|+\left\|\widehat{\boldsymbol{\psi}}_{N}^{h}-\boldsymbol{\psi}_{N}^{h}\right\| \\
& \leq c(\Delta t+h)+\left\|\widehat{\boldsymbol{\psi}}_{N}^{h}-\boldsymbol{\psi}_{N}^{h}\right\|,
\end{aligned}
$$

where $\boldsymbol{\psi}_{N}$ and $\widehat{\boldsymbol{\psi}}_{N}^{h}$ are defined as in (3.22) and (3.24), respectively. For simplicity of the notation, we abbreviate $\widehat{\boldsymbol{\psi}}_{N}^{h}\left(t_{n}, \mathbf{x}\right)$ and $\boldsymbol{\psi}_{N}^{h}\left(t_{n}, \mathbf{x}\right)$ to $\widehat{\boldsymbol{\psi}}_{n}^{h}(\mathbf{x})$ and $\boldsymbol{\psi}_{n}^{h}(\mathbf{x})$, respectively. The definitions of $\widehat{\boldsymbol{\psi}}_{n}^{h}$ and $\boldsymbol{\psi}_{n}^{h}$ and the triangle inequality imply

$$
\left\|\widehat{\boldsymbol{\psi}}_{n-1}^{h}-\boldsymbol{\psi}_{n-1}^{h}\right\| \leq\left\|\widehat{\boldsymbol{\psi}}_{n}^{h}-\boldsymbol{\psi}_{n}^{h}\right\|+\Delta t\left\|\nabla_{\boldsymbol{\phi}} \widehat{\mathbf{u}}^{h}\left(\widehat{\boldsymbol{\phi}}_{n-1}^{h}\right) \widehat{\boldsymbol{\psi}}_{n}^{h}-\nabla_{\boldsymbol{\phi}} \mathbf{u}^{h}\left(\boldsymbol{\phi}_{n-1}^{h}\right) \boldsymbol{\psi}_{n}^{h}\right\| .
$$

By the triangle inequality and the change of variables (3.60), we have

$$
\begin{aligned}
& \left\|\nabla_{\boldsymbol{\phi}} \widehat{\mathbf{u}}^{h}\left(\widehat{\boldsymbol{\phi}}_{n-1}^{h}\right) \widehat{\boldsymbol{\psi}}_{n}^{h}-\nabla_{\boldsymbol{\phi}} \mathbf{u}^{h}\left(\boldsymbol{\phi}_{n-1}^{h}\right) \boldsymbol{\psi}_{n}^{h}\right\| \\
& \leq\left\|\nabla_{\boldsymbol{\phi}} \widehat{\mathbf{u}}^{h}\left(\widehat{\boldsymbol{\phi}}_{n-1}^{h}\right) \widehat{\boldsymbol{\psi}}_{n}^{h}-\nabla_{\boldsymbol{\phi}} \widehat{\mathbf{u}}^{h}\left(\boldsymbol{\phi}_{n-1}^{h}\right) \widehat{\boldsymbol{\psi}}_{n}^{h}\right\|+ \\
& \left\|\nabla_{\boldsymbol{\phi}} \widehat{\mathbf{u}}^{h}\left(\boldsymbol{\phi}_{n-1}^{h}\right) \widehat{\boldsymbol{\psi}}_{n}^{h}-\nabla_{\boldsymbol{\phi}} \widehat{\mathbf{u}}^{h}\left(\boldsymbol{\phi}_{n-1}^{h}\right) \boldsymbol{\psi}_{n}^{h}\right\|+\left\|\nabla_{\boldsymbol{\phi}} \widehat{\mathbf{u}}^{h}\left(\boldsymbol{\phi}_{n-1}^{h}\right) \boldsymbol{\psi}_{n}^{h}-\nabla_{\boldsymbol{\phi}} \mathbf{u}^{h}\left(\boldsymbol{\phi}_{n-1}^{h}\right) \boldsymbol{\psi}_{n}^{h}\right\| \\
& \leq c\left\|\nabla \widehat{\mathbf{u}}^{h}\right\|_{\infty}\left\|\widehat{\boldsymbol{\psi}}_{n}^{h}\right\|_{\infty}\left\|\widehat{\boldsymbol{\phi}}_{n-1}^{h}-\boldsymbol{\phi}_{n-1}^{h}\right\| \\
& \quad+\left\|\nabla \widehat{\mathbf{u}}^{h}\right\|_{\infty}\left\|\widehat{\boldsymbol{\psi}}_{n}^{h}-\boldsymbol{\psi}_{n}^{h}\right\|+\left\|\boldsymbol{\psi}_{n}^{h}\right\|_{\infty} \gamma^{\frac{n-1}{2}}\left\|\nabla \widehat{\mathbf{u}}^{h}-\nabla \mathbf{u}^{h}\right\|,
\end{aligned}
$$

where $\gamma=1 /\left|2-\Delta t\left\|\nabla \cdot \mathbf{u}^{h}\right\|_{\infty}\right|$. Again, we use the inequality (3.17) to obtain

$$
\begin{aligned}
& \left\|\widehat{\boldsymbol{\psi}}_{n}^{h}\right\|_{\infty} \leq\left\|\widehat{\boldsymbol{\psi}}_{n+1}^{h}\right\|_{\infty}+2 \Delta t\left\|\nabla \widehat{\mathbf{u}}^{h}\right\|_{\infty}\left\|\widehat{\boldsymbol{\psi}}_{n+1}^{h}\right\|_{\infty}=\left(1+2 \Delta t\left\|\nabla \widehat{\mathbf{u}}^{h}\right\|_{\infty}\right)\left\|\widehat{\boldsymbol{\psi}}_{n+1}^{h}\right\|_{\infty} \\
& \cdots \leq\left(1+2 \Delta t\left\|\nabla \widehat{\mathbf{u}}^{h}\right\|_{\infty}\right)^{N-n}\left\|\widehat{\boldsymbol{\psi}}_{N}^{h}\right\|_{\infty} \leq\left(1+2 c_{0} \Delta t\right)^{N-n} C_{t r} \leq e^{2 c_{0}} C_{t r}
\end{aligned}
$$

where $c_{0}=c\|\mathbf{u}\|_{2}+\|\nabla \mathbf{u}\|_{\infty}$ and $C_{t r} \equiv\left\|\widehat{\boldsymbol{\psi}}_{N}^{h}\right\|_{\infty} \leq c\left(\|\mathbf{T}\|_{2}^{2}+\|\mathbf{T}\|_{1, \infty}^{2}+\|\mathbf{R}\|_{2}^{2}+\|\mathbf{R}\|_{\infty}^{2}\right)$, under the assumption $\Delta t \leq 1 /\left(2 c_{0}\right)$. The inverse inequality, $\|\cdot\|_{\infty} \leq c_{I} h^{-1}\|\cdot\|$, and the change of variables (3.60) yield

$$
\begin{aligned}
\left\|\boldsymbol{\psi}_{n}^{h}\right\|_{\infty} & \leq\left\|\boldsymbol{\psi}_{n+1}^{h}\right\|_{\infty}+2 \Delta t\left\|\nabla_{\boldsymbol{\phi}} \mathbf{u}^{h}\left(\boldsymbol{\phi}_{n}^{h}\right)\right\|_{\infty}\left\|\boldsymbol{\psi}_{n+1}^{h}\right\|_{\infty} \\
& \leq\left(1+2 c_{I} \Delta t h^{-1}\left\|\nabla_{\boldsymbol{\phi}} \mathbf{u}^{h}\left(\boldsymbol{\phi}_{n}^{h}\right)\right\|\right)\left\|\boldsymbol{\psi}_{n+1}^{h}\right\|_{\infty} \\
& \leq\left(1+2 c_{I} \Delta t h^{-1}\left\|\nabla \mathbf{u}^{h}\right\| \gamma^{\frac{k}{2}}\right)\left\|\boldsymbol{\psi}_{n+1}^{h}\right\|_{\infty} \\
& \leq \prod_{m=n}^{N-1}\left(1+2 c_{I} \Delta t h^{-1}\left\|\nabla \mathbf{u}^{h}\right\| \gamma^{\frac{m}{2}}\right) C_{t r} .
\end{aligned}
$$

Suppose that we choose $\Delta t$ such that

$$
\Delta t \leq \frac{\epsilon^{2}+2 \epsilon-1}{(1+\epsilon)^{2}} \min \left\{\frac{1}{\left\|\nabla \cdot \mathbf{u}^{h}\right\|_{\infty}}, \frac{h}{2 c_{I}\left\|\nabla \mathbf{u}^{h}\right\|}, \frac{1}{2 c_{0}}\right\}
$$

for all constant $\epsilon$ in $\left(\sqrt{2}-1, \frac{\sqrt{5}-1}{2}\right] \sim(0.4142,0.6810]$. Then,

$$
\gamma \leq\left(\frac{1+\epsilon}{2}\right)^{2}<1 \quad \text { and } \quad 2 c_{I} \Delta t h^{-1}\left\|\nabla \mathbf{u}^{h}\right\| \leq \frac{\epsilon^{2}+2 \epsilon-1}{(1+\epsilon)^{2}} \leq \frac{1-\epsilon}{1+\epsilon} .
$$


If $0<j<1$, then $1+j<e^{j}$ and this yields

$$
\begin{gathered}
\prod_{m=n}^{N-1}\left(1+2 c_{I} \Delta t h^{-1}\left\|\nabla \mathbf{u}^{h}\right\| \gamma^{\frac{m}{2}}\right) \leq \prod_{m=1}^{N}\left(1+\frac{1-\epsilon}{1+\epsilon} \cdot\left(\frac{1+\epsilon}{2}\right)^{m}\right) \\
\leq \prod_{m=1}^{N} e^{\frac{1-\epsilon}{1+\epsilon} \cdot\left(\frac{1+\epsilon}{2}\right)^{m}}=e^{\frac{1-\epsilon}{1+\epsilon} \cdot\left(\left(\frac{1+\epsilon}{2}\right)+\left(\frac{1+\epsilon}{2}\right)^{2}+\cdots+\left(\frac{1+\epsilon}{2}\right)^{N}\right) \leq e}
\end{gathered}
$$

Now, gather (3.63)-(3.65) and (3.67) and then apply (3.17) to obtain

$$
\begin{gathered}
\left\|\nabla_{\boldsymbol{\phi}} \widehat{\mathbf{u}}^{h}\left(\widehat{\boldsymbol{\phi}}_{n-1}^{h}\right) \widehat{\boldsymbol{\psi}}_{n}^{h}-\nabla_{\boldsymbol{\phi}} \mathbf{u}^{h}\left(\boldsymbol{\phi}_{n-1}^{h}\right) \boldsymbol{\psi}_{n}^{h}\right\| \\
\leq c_{0}\left(c e^{2 c_{0}} C_{t r}\left\|\widehat{\boldsymbol{\phi}}_{n-1}^{h}-\boldsymbol{\phi}_{n-1}^{h}\right\|+\left\|\widehat{\boldsymbol{\psi}}_{n}^{h}-\boldsymbol{\psi}_{n}^{h}\right\|\right)+e C_{t r} \gamma^{\frac{n-1}{2}}\left\|\nabla \widehat{\mathbf{u}}^{h}-\nabla \mathbf{u}^{h}\right\| \\
\leq c\left\|\widehat{\boldsymbol{\phi}}_{n-1}^{h}-\boldsymbol{\phi}_{n-1}^{h}\right\|+c\left\|\widehat{\boldsymbol{\psi}}_{n}^{h}-\boldsymbol{\psi}_{n}^{h}\right\|+c \gamma^{\frac{n-1}{2}}\left\|\nabla \widehat{\mathbf{u}}^{h}-\nabla \mathbf{u}^{h}\right\| .
\end{gathered}
$$

Hence, we have

$$
\begin{aligned}
& \left\|\widehat{\boldsymbol{\psi}}_{n-1}^{h}-\boldsymbol{\psi}_{n-1}^{h}\right\| \\
& \leq(1+c \Delta t)\left\|\widehat{\boldsymbol{\psi}}_{n}^{h}-\boldsymbol{\psi}_{n}^{h}\right\|+c \Delta t\left\|\widehat{\boldsymbol{\phi}}_{n-1}^{h}-\boldsymbol{\phi}_{n-1}^{h}\right\|+c \Delta t \gamma^{\frac{n-1}{2}}\left\|\nabla \widehat{\mathbf{u}}^{h}-\nabla \mathbf{u}^{h}\right\| \\
& \quad \cdots \leq(1+c \Delta t)^{N-n+1}\left\|\widehat{\boldsymbol{\psi}}_{N}^{h}-\boldsymbol{\psi}_{N}^{h}\right\|+c \Delta t \sum_{i=n}^{N}(1+c \Delta t)^{i-n}\left\|\widehat{\boldsymbol{\phi}}_{i-1}^{h}-\boldsymbol{\phi}_{i-1}^{h}\right\| \\
& \quad+c \Delta t\left\|\nabla \widehat{\mathbf{u}}^{h}-\nabla \mathbf{u}^{h}\right\| \sum_{j=n}^{N}(1+c \Delta t)^{j-n} \gamma^{\frac{j-1}{2}} \\
& \leq c e^{c} \Delta t \sum_{i=n}^{N}\left\|\widehat{\boldsymbol{\phi}}_{i-1}^{h}-\boldsymbol{\phi}_{i-1}^{h}\right\|+c e^{c} \Delta t\left\|\nabla \widehat{\mathbf{u}}^{h}-\nabla \mathbf{u}^{h}\right\| \sum_{j=n}^{N} \gamma^{\frac{j-1}{2}},
\end{aligned}
$$

where $\widehat{\boldsymbol{\psi}}_{N}^{h}-\boldsymbol{\psi}_{N}^{h}=\mathbf{0}$ and $(1+c \Delta t)^{n} \leq e^{c n \Delta t}=e^{c \frac{n}{N}}$ with $n \leq N$. From (3.61), we can calculate

$$
\begin{aligned}
\sum_{i=n}^{N}\left\|\widehat{\boldsymbol{\phi}}_{i-1}^{h}-\boldsymbol{\phi}_{i-1}^{h}\right\|^{2} & \leq c \Delta t\left\|\widehat{\mathbf{u}}^{h}-\mathbf{u}^{h}\right\|^{2} \sum_{i=n}^{N}\left(1+2 c_{0} \Delta t\right)^{i-1} \\
& \leq c\left\|\widehat{\mathbf{u}}^{h}-\mathbf{u}^{h}\right\|^{2}\left(1+2 c_{0} \Delta t\right)^{n-1}
\end{aligned}
$$

Because $\sum_{j=n}^{N} \gamma^{\frac{j-1}{2}} \leq \gamma^{\frac{n-1}{2}} \sum_{j=n}^{N}\left(\frac{1+\epsilon}{2}\right)^{j-n} \leq \gamma^{\frac{n-1}{2}} \frac{2}{1-\epsilon} \leq \gamma^{\frac{n-1}{2}}(2+\sqrt{2})$, the inequalities (3.68), (3.69) and Proposition 3.9 imply

$$
\begin{aligned}
\left\|\widehat{\boldsymbol{\psi}}_{N}^{h}-\boldsymbol{\psi}_{N}^{h}\right\|^{2} & =\Delta t \sum_{n=0}^{N}\left\|\widehat{\boldsymbol{\psi}}_{N}^{h}\left(t_{n}, \mathbf{x}\right)-\boldsymbol{\psi}_{N}^{h}\left(t_{n}, \mathbf{x}\right)\right\|^{2}=\Delta t \sum_{n=0}^{N}\left\|\widehat{\boldsymbol{\psi}}_{n}^{h}-\boldsymbol{\psi}_{n}^{h}\right\|^{2} \\
& \leq c \Delta t^{3}\left\|\widehat{\mathbf{u}}^{h}-\mathbf{u}^{h}\right\|^{2} \sum_{n=0}^{N}\left(1+2 c_{0} \Delta t\right)^{n}+c \Delta t^{2}\left\|\nabla \widehat{\mathbf{u}}^{h}-\nabla \mathbf{u}^{h}\right\|^{2} \\
& \leq c \Delta t^{2}\left\|\widehat{\mathbf{u}}^{h}-\mathbf{u}^{h}\right\|_{1}^{2} .
\end{aligned}
$$

Proposition 3.11. Let $\mathbf{F}$ and $\mathbf{F}_{N}^{h}$ be defined as in (3.41) and (3.56), respectively. If $(\eta, \xi)^{t}$ and $\left(\eta^{h}, \xi^{h}\right)^{t}$ satisfy (3.41) and (3.56), respectively, then we have

$$
\left\|\eta-\eta^{h}\right\|+\left\|\xi-\xi^{h}\right\| \leq c(h+\Delta t)+c \sqrt{\Delta t}\left\|\boldsymbol{\psi}_{N}-\boldsymbol{\psi}_{N}^{h}\right\| .
$$


Proof. For a given $\mathbf{F}=\int_{I}\left|\operatorname{det} \nabla \boldsymbol{\phi}^{-1}(t, \mathbf{x})\right| \boldsymbol{\psi}\left(t, \boldsymbol{\phi}^{-1}(t, \mathbf{x})\right) d t,(\eta, \xi)^{t}$ satisfies $\nabla^{\perp} \eta-$ $\nabla \xi=\mathbf{F}$. As shown in Section 3.1.4, we define $\left(\widehat{\eta}^{h}, \widehat{\xi}^{h}\right)^{t}=\mathcal{L}^{*} \widehat{\mathbf{w}}^{h}$, where $\widehat{\mathbf{w}}^{h}$ is a solution of (3.46), i.e.,

$$
\left\langle\mathcal{L}^{*} \widehat{\mathbf{w}}^{h}, \mathcal{L}^{*} \mathbf{v}^{h}\right\rangle=\left\langle(\eta, \xi)^{t}, \mathcal{L}^{*} \mathbf{v}^{h}\right\rangle=\left\langle\mathcal{L}(\eta, \xi)^{t}, \mathbf{v}^{h}\right\rangle=\left\langle\mathbf{F}, \mathbf{v}^{h}\right\rangle
$$

for all $\mathbf{v}^{h} \in \mathcal{V}^{h}$.

Also, for a given $\mathbf{F}_{N}^{h}=\Delta t \sum_{n=0}^{N}\left|\operatorname{det} \nabla \boldsymbol{\phi}_{N}^{-1}\left(t_{n}, \mathbf{x}\right)\right| \boldsymbol{\psi}_{N}^{h}\left(t_{n}, \boldsymbol{\phi}_{N}^{-1}\left(t_{n}, \mathbf{x}\right)\right)$ with $\boldsymbol{\phi}_{N}$ from (3.6), there exists a $(\eta \underline{\eta}, \underline{\xi})^{t}$ satisfying $\nabla^{\perp} \underline{\eta}-\nabla \underline{\xi}=\mathbf{F}_{N}^{h}$. From (3.56), we let $\left(\eta^{h}, \xi^{h}\right)^{t}$ be defined as $\mathcal{L}^{*} \mathbf{w}^{h}$ which is a solution of the weak problem

$$
\left\langle\mathcal{L}^{*} \mathbf{w}^{h}, \mathcal{L}^{*} \mathbf{v}^{h}\right\rangle=\left\langle(\underline{\eta}, \underline{\xi})^{t}, \mathcal{L}^{*} \mathbf{v}^{h}\right\rangle=\left\langle\mathbf{F}_{N}^{h}, \mathbf{v}^{h}\right\rangle
$$

for all $\mathbf{v}^{h} \in \mathcal{V}^{h}$. Now, subtracting (3.71) from (3.70) yields

$$
\left\langle\mathcal{L}^{*}\left(\widehat{\mathbf{w}}^{h}-\mathbf{w}^{h}\right), \mathcal{L}^{*} \mathbf{v}^{h}\right\rangle=\left\langle\mathbf{F}-\mathbf{F}_{N}^{h}, \mathbf{v}^{h}\right\rangle, \text { for } \forall \mathbf{v}^{h} \in \mathcal{V}^{h} .
$$

Let $\mathbf{v}^{h}=\widehat{\mathbf{w}}^{h}-\mathbf{w}^{h}$. Because $\widehat{\mathbf{w}}^{h}-\mathbf{w}^{h} \in H_{N}(\nabla \cdot) \cap H(\nabla \times)$ (see [9]),

$$
\left\|\widehat{\mathbf{w}}^{h}-\mathbf{w}^{h}\right\|^{2} \leq c\left(\left\|\nabla \cdot\left(\widehat{\mathbf{w}}^{h}-\mathbf{w}^{h}\right)\right\|^{2}+\left\|\nabla \times\left(\widehat{\mathbf{w}}^{h}-\mathbf{w}^{h}\right)\right\|^{2}\right)
$$

which implies

$$
\left\|\left(\widehat{\eta}^{h}, \widehat{\xi}^{h}\right)-\left(\eta^{h}, \xi^{h}\right)\right\|=\left\|\mathcal{L}^{*} \widehat{\mathbf{w}}^{h}-\mathcal{L}^{*} \mathbf{w}^{h}\right\| \leq c\left\|\mathbf{F}-\mathbf{F}_{N}^{h}\right\| .
$$

By the triangle inequality and Proposition 3.6, we have

$$
\left\|(\eta, \xi)-\left(\eta^{h}, \xi^{h}\right)\right\| \leq\left\|(\eta, \xi)-\left(\widehat{\eta}^{h}, \widehat{\xi}^{h}\right)\right\|+\left\|\left(\widehat{\eta}^{h}, \widehat{\xi}^{h}\right)-\left(\eta^{h}, \xi^{h}\right)\right\| \leq c h+c\left\|\mathbf{F}-\mathbf{F}_{N}^{h}\right\| .
$$

Now, define $\mathbf{F}_{N}=\Delta t \sum_{n=0}^{N}\left|\operatorname{det} \nabla \boldsymbol{\phi}_{N}^{-1}\left(t_{n}, \mathbf{x}\right)\right| \boldsymbol{\psi}_{N}\left(t_{n}, \boldsymbol{\phi}_{N}^{-1}\left(t_{n}, \mathbf{x}\right)\right)$. By the triangle inequality,

$$
\left\|\mathbf{F}-\mathbf{F}_{N}^{h}\right\| \leq\left\|\mathbf{F}-\mathbf{F}_{N}\right\|+\left\|\mathbf{F}_{N}-\mathbf{F}_{N}^{h}\right\| \leq c \Delta t+\left\|\mathbf{F}_{N}-\mathbf{F}_{N}^{h}\right\| .
$$

Therefore, the change of variables (3.21) yields

$$
\begin{aligned}
& \left\|\mathbf{F}_{N}-\mathbf{F}_{N}^{h}\right\|^{2} \\
& \leq 2 \Delta t^{2} \sum_{n=0}^{N} \int_{\Omega}\left|\operatorname{det} \nabla \boldsymbol{\phi}_{N}^{-1}\left(t_{n}, \mathbf{x}\right)\right|^{2}\left|\boldsymbol{\psi}_{N}\left(t_{n}, \boldsymbol{\phi}_{N}^{-1}\left(t_{n}, \mathbf{x}\right)\right)-\boldsymbol{\psi}_{N}^{h}\left(t_{n}, \boldsymbol{\phi}_{N}^{-1}\left(t_{n}, \mathbf{x}\right)\right)\right|^{2} d \mathbf{x} \\
& \leq c \Delta t^{2} \sum_{n=0}^{N} \int_{\Omega}\left|\boldsymbol{\psi}_{N}\left(t_{n}, \mathbf{y}\right)-\boldsymbol{\psi}_{N}^{h}\left(t_{n}, \mathbf{y}\right)\right|^{2} d \mathbf{y}=c \Delta t\left\|\boldsymbol{\psi}_{N}-\boldsymbol{\psi}_{N}^{h}\right\|^{2}
\end{aligned}
$$

under the assumption $\Delta t \leq 1 /\|\nabla \cdot \mathbf{u}\|_{\infty} \cdot \mathbf{Q}$

Proposition 3.12. Let $(\mathbf{p}, f),(\mathbf{q}, g),\left(\mathbf{p}^{h}, f^{h}\right)$, and $\left(\mathbf{q}^{h}, g^{h}\right)$ be the solutions of (3.49), (3.50), (3.57), and (3.58), respectively. Then, we have

$$
\left\|f-f^{h}\right\|_{1}+\left\|g-g^{h}\right\|_{1} \leq c\left(\left\|\xi-\xi^{h}\right\|+\left\|\eta-\eta^{h}\right\|\right)
$$


Proof. First, we focus on $f$. Let $\left(\widehat{\mathbf{p}}^{h}, \widehat{f}^{h}\right)$ satisfy (3.51), Then, we have

$$
\left\|f-f^{h}\right\|_{1} \leq\left\|f-\widehat{f}^{h}\right\|_{1}+\left\|\widehat{f}^{h}-f^{h}\right\|_{1} .
$$

Subtracting (3.57) from (3.51) implies

$$
\begin{gathered}
\left\langle\widehat{\mathbf{p}}^{h}-\mathbf{p}^{h}-\nabla\left(\widehat{f}^{h}-f^{h}\right), \mathbf{r}^{h}-\nabla r^{h}\right\rangle+\frac{1}{\beta_{f}^{2}}\left\langle\nabla \times\left(\widehat{\mathbf{p}}^{h}-\mathbf{p}^{h}\right), \nabla \times \mathbf{r}^{h}\right\rangle \\
\quad+\left\langle-\frac{1}{\beta_{f}} \nabla \cdot\left(\widehat{\mathbf{p}}^{h}-\mathbf{p}^{h}\right)+\beta_{f}\left(\widehat{f}^{h}-f^{h}\right),-\frac{1}{\beta_{f}} \nabla \cdot \mathbf{r}^{h}+\beta_{f} r^{h}\right\rangle \\
=\left\langle-\frac{1}{\alpha_{f_{1}} \beta_{f}}\left(\sigma-\sigma^{h}-\xi+\xi^{h}\right),-\frac{1}{\beta_{f}} \nabla \cdot \mathbf{r}^{h}+\beta_{f} r^{h}\right\rangle
\end{gathered}
$$

for all $\left(r^{h}, \mathbf{r}^{h}\right) \in \mathcal{X}^{h} \times X^{h}$. We let $r^{h}=\widehat{f}^{h}-f^{h}$ and $\mathbf{r}^{h}=\widehat{\mathbf{p}}^{h}-\mathbf{p}^{h}$ in the above and use the Hölder inequality, the $\epsilon$-inequality, and Green's formula to obtain

$$
\left\|\widehat{\mathbf{p}}^{h}-\mathbf{p}^{h}\right\|_{1}^{2}+\left\|\widehat{f}^{h}-f^{h}\right\|_{1}^{2} \leq c\left(\left\|\sigma-\sigma^{h}\right\|^{2}+\left\|\xi-\xi^{h}\right\|^{2}\right) \leq c\left\|\xi-\xi^{h}\right\|^{2} .
$$

The last inequality results from

$$
\left\|\sigma-\sigma^{h}\right\|^{2}=\frac{1}{|\Omega|^{2}} \int_{\Omega}\left(\int_{I} \xi d t-\int_{I} \xi^{h} d t\right)^{2} d \mathbf{x} \leq c\left\|\xi-\xi^{h}\right\|^{2} .
$$

Analogously, we have

$$
\left\|\widehat{\mathbf{q}}^{h}-\mathbf{q}^{h}\right\|_{1}^{2}+\left\|\widehat{g}^{h}-g^{h}\right\|_{1}^{2} \leq c\left\|\eta-\eta^{h}\right\|^{2} .
$$

Finally, we gather Propositions 3.8-3.12 to obtain the following result.

TheOrem 3.13. Let $(\mathbf{u}, \boldsymbol{\phi}, \boldsymbol{\psi}, \xi, \eta, f, g)$ denote the solution of the optimality system (2.4)-(2.8) and $\left(\mathbf{u}^{h}, \boldsymbol{\phi}_{N}^{h}, \boldsymbol{\psi}_{N}^{h}, \xi^{h}, \eta^{h}, f^{h}, g^{h}\right) \in \mathcal{U}^{h} \times\left(\Phi^{h}\right)^{2} \times \mathcal{V}^{h} \times \mathcal{X}^{h} \times \mathcal{Y}^{h}$ the solution of the fully discretized optimality system (3.53)-(3.58). Suppose that $\Delta t$ is sufficiently small. Then,

$$
\begin{gathered}
\left\|\mathbf{u}-\mathbf{u}^{h}\right\|_{1}^{2}+\left\|\boldsymbol{\phi}-\boldsymbol{\phi}^{h}\right\|^{2}+\left\|\boldsymbol{\psi}-\boldsymbol{\psi}^{h}\right\|^{2}+\left\|\xi-\xi^{h}\right\|+\left\|\eta-\eta^{h}\right\|^{2} \\
+\left\|f-f^{h}\right\|_{1}^{2}+\left\|g-g^{h}\right\|_{1}^{2} \leq c\left(\Delta t^{2}+h^{2}\right) .
\end{gathered}
$$

Proof. Theorems 3.8-3.12 yield

$$
\begin{gathered}
\left\|\mathbf{u}-\mathbf{u}^{h}\right\|_{1}^{2}+\left\|\boldsymbol{\phi}-\boldsymbol{\phi}^{h}\right\|^{2}+\left\|\boldsymbol{\psi}-\boldsymbol{\psi}^{h}\right\|^{2}+\left\|\xi-\xi^{h}\right\|+\left\|\eta-\eta^{h}\right\|^{2} \\
+\left\|f-f^{h}\right\|_{1}^{2}+\left\|g-g^{h}\right\|_{1}^{2} \leq c\left(\Delta t^{2}+h^{2}\right)+c \Delta t\left\|\mathbf{u}-\mathbf{u}^{h}\right\|_{1}^{2}
\end{gathered}
$$

where $c$ does not depend on $\Delta t$ and $h$. Hence, for sufficiently small $\Delta t$, the result holds.

4. Gradient algorithm. In Section 3, we proved that the solution of the discrete optimality system (3.53)-(3.57) converges to the solution of the optimality system (2.4)-(2.8). Due to the forward-in-time nature of the state equations and the backward-in-time nature of the adjoint equations, solving the fully-coupled discrete optimality system is a formidable task. In most cases, it is more efficient to solve the optimality system using an optimization iteration for which, at each iteration, the different components of the discrete optimality system are solved sequentially. Here, we consider a gradient method for this purpose. 
The discrete functional used in the optimal control problem is defined as

$$
\begin{aligned}
& J_{h}\left(\left.\phi^{h}\right|_{t=1}, f^{h}, g^{h}\right)=\frac{1}{2}\left\|\mathbf{T}^{h}\left(\phi^{h}(1, \mathbf{x})\right)-\mathbf{R}^{h}\right\|^{2} \\
& \quad+\frac{\alpha_{f_{0}}}{2}\left\|f^{h}\right\|^{2}+\frac{\alpha_{f_{1}}}{2}\left\|\nabla f^{h}\right\|^{2}+\frac{\alpha_{g_{0}}}{2}\left\|g^{h}\right\|^{2}+\frac{\alpha_{g_{1}}}{2}\left\|\nabla g^{h}\right\|^{2}-\beta \int_{\Omega} \log f^{h} d \mathbf{x},
\end{aligned}
$$

where $\mathbf{T}^{h} \equiv \mathrm{I}^{h} \mathbf{T}$ and $\mathbf{R}^{h} \equiv \mathrm{I}^{h} \mathbf{R}$. Here, for notational simplicity, we omit the subscript $N$ that represents the number of time steps used in the integrations of $\boldsymbol{\phi}^{h}$ and $\boldsymbol{\psi}^{h}$. However, throughout this section, we still have $\left\{t_{n}\right\}_{n=0}^{N}$ as a partition of $[0,1]$ into equal intervals $\Delta t=1 / N$ with $t_{0}=0$ and $t_{N}=1$.

Let $J_{h}(m)=J_{h}\left(\phi^{h}(m), f^{h}(m), g^{h}(m)\right)$, where $m$ is the iteration counter of the gradient algorithm and $\phi^{h}(m)$ presents $\phi^{h}(1, \mathbf{x})$ at the $m$-th iterative step. In the algorithm, $\tau$ denotes a prescribed tolerance used to test for the convergence of the functional, $s$ denotes a step-size parameter that is automatically selected by the algorithm, and $\left(f^{h}(0), g^{h}(0)\right)$ denotes an initial guess for the controls.

The gradient algorithm proceeds as follows [10].

(a) initialization :

(i) choose $\tau$ and $\left(f^{h}(0), g^{h}(0)\right)$; set $m=0$ and $s=1$;

(ii) solve for $\mathbf{u}^{h}(0)$ and then $\boldsymbol{\phi}^{h}(0)$ from (3.53) and (3.54), respectively with $\left(f^{h}, g^{h}\right)=\left(f^{h}(0), g^{h}(0)\right)$

(iii) evaluate $J_{h}(0)$;

(b) main loop;

(iv) set $m=m+1$;

(v) solve for $\boldsymbol{\psi}^{h}(m)$ from (3.55), $\left(\eta^{h}(m), \xi^{h}(m)\right)$ from $(3.56),\left(f^{h}(m), g^{h}(m)\right)$ from (3.57) and (3.58), respectively, with $\mathbf{u}^{h}=\mathbf{u}^{h}(m-1)$ and $\phi^{h}=$ $\phi^{h}(m-1)$

(vi) set

$$
\begin{aligned}
f^{h}(m)= & f^{h}(m-1) \\
& -s\left(-\alpha_{f_{1}} \Delta\left(f^{h}(m-1)-f^{h}(m)\right)+\alpha_{f_{0}}\left(f^{h}(m-1)-f^{h}(m)\right)\right) \\
g^{h}(m)= & g^{h}(m-1) \\
& -s\left(-\alpha_{g_{1}} \Delta\left(g^{h}(m-1)-g^{h}(m)\right)+\alpha_{g_{0}}\left(g^{h}(m-1)-g^{h}(m)\right)\right)
\end{aligned}
$$

(vii) solve for $\mathbf{u}^{h}(m)$ and then $\phi^{h}(m)$ from (3.53) and (3.54), respectively with $\left(f^{h}, g^{h}\right)=\left(f^{h}(m), g^{h}(m)\right)$;

(viii) evaluate $J_{h}(m)=J_{h}\left(\phi^{h}(m), f^{h}(m), g^{h}(m)\right)$;

(ix) if $J_{h}(m) \geq J_{h}(m-1)$, set $s=0.5 s$ and go to step (vi); otherwise, continue;

(x) if $\left|J_{h}(m)-J_{h}(m-1)\right| /\left|J_{h}(m)\right|>\tau$, set $s=1.5 s$ and go to step (iv); otherwise, stop;

The convergence of gradient algorithm is shown in the following theorem.

TheOREM 4.1. Let $\left(\mathbf{u}^{h}(m), \boldsymbol{\phi}^{h}(m), \boldsymbol{\psi}^{h}(m), \eta^{h}(m), \xi^{h}(m), f^{h}(m), g^{h}(m)\right)$ denote the $m$-th iterate of the gradient algorithm. Let $\left(\mathbf{u}^{h}, \boldsymbol{\phi}^{h}, \boldsymbol{\psi}^{h}, \eta^{h}, \xi^{h}, f^{h}, g^{h}\right)$ denote the solution of the discrete optimality system (3.53)-(3.57). Then, for $\Delta t$ sufficiently small, there exists a ball $B$ in $\left[X^{h}\right]^{2}$ whose radius depends on the penalty parameters, $\alpha_{f_{0}}, \alpha_{f_{1}}, \alpha_{g_{0}}, \alpha_{g_{1}}$, such that, if $\left(f^{h}(0), g^{h}(0)\right) \in B$, then

$$
\begin{array}{r}
\left(\mathbf{u}^{h}(m), \boldsymbol{\phi}^{h}(m), \boldsymbol{\psi}^{h}(m), \eta^{h}(m), \xi^{h}(m), f^{h}(m), g^{h}(k)\right) \\
\rightarrow\left(\mathbf{u}^{h}, \boldsymbol{\phi}^{h}, \boldsymbol{\psi}^{h}, \eta^{h}, \xi^{h}, f^{h}, g^{h}\right) \quad \text { as } m \rightarrow \infty .
\end{array}
$$


Proof. Recall the classical result in [6]: Let $V$ be a Hilbert space and let $J: V \rightarrow \mathbb{R}$ be a functional of class $\mathcal{C}^{2}$ which has a local minimum at a point $q \in V$. Suppose that there are two constants $c_{1}$ and $c_{2}$ and a ball $B \subset V$ centered at $g$ satisfying, for all $\delta g_{1}, \delta g_{2} \in V$ and any $\widetilde{g} \in B$,

$$
D^{2} J(\widetilde{g})\left(\delta g_{1}, \delta g_{2}\right) \leq c_{1}\left\|\delta g_{1}\right\|\left\|\delta g_{2}\right\| \quad \text { and } \quad D^{2} J(\widetilde{g})\left(\delta g_{1}, \delta g_{1}\right) \geq c_{2}\left\|\delta g_{1}\right\|^{2},
$$

where $D^{2} J$ is the second Frechét derivative of $J$. Then, for all $g_{0} \in B$, the iterates of the gradient algorithm converge to $g$.

For each $\left(f^{h}, g^{h}\right) \in\left(X^{h}\right)^{2}$, the second Frechét derivative of $J^{h}$ is given by

$$
\begin{aligned}
& D^{2} J^{h}\left(\boldsymbol{\phi}_{N}^{h} ; f^{h}, g^{h}\right)\left[\left(\widetilde{f}^{h}, \widetilde{g}^{h}\right),\left(\widetilde{\widetilde{f}}^{h}, \widetilde{\widetilde{g}}^{h}\right)\right] \\
& =\int_{\Omega} \widehat{\boldsymbol{\phi}}_{N}^{h}(\mathbf{x}) \cdot \nabla_{\boldsymbol{\phi}} \mathbf{T}^{h}\left(\mathbf{T}^{h}\left(\boldsymbol{\phi}_{N}^{h}(\mathbf{x})\right)-\mathbf{R}^{h}\right) d \mathbf{x} \\
& +\int_{\Omega} \widetilde{\boldsymbol{\phi}}_{N}^{h}(\mathbf{x}) \cdot\left(\widetilde{\widetilde{\phi}}_{N}^{h}(\mathbf{x}) \cdot \nabla_{\boldsymbol{\phi}} \nabla_{\boldsymbol{\phi}} \mathbf{T}^{h}\left(\boldsymbol{\phi}_{N}^{h}(x)\right)\right)\left(\mathbf{T}^{h}\left(\boldsymbol{\phi}_{N}^{h}(\mathbf{x})\right)-\mathbf{R}^{h}\right) d \mathbf{x} \\
& +\int_{\Omega}\left(\widetilde{\boldsymbol{\phi}}_{N}^{h}(\mathbf{x}) \cdot \nabla_{\boldsymbol{\phi}} \mathbf{T}^{h}\left(\boldsymbol{\phi}_{N}^{h}(\mathbf{x})\right)\right)\left(\widetilde{\widetilde{\phi}}_{N}^{h}(\mathbf{x}) \cdot \nabla_{\boldsymbol{\phi}} \mathbf{T}^{h}\left(\boldsymbol{\phi}_{N}^{h}(\mathbf{x})\right)\right) d \mathbf{x} \\
& +\int_{\Omega} \alpha_{f_{0}} \widetilde{f}^{h} \widetilde{\widetilde{f}}^{h}+\alpha_{f_{1}} \nabla \widetilde{f}^{h} \cdot \nabla \widetilde{\widetilde{f}}^{h}+\alpha_{g_{0}} \widetilde{g}^{h} \widetilde{\widetilde{g}}^{h}+\alpha_{g_{1}} \nabla \widetilde{g}^{h} \cdot \nabla \widetilde{\widetilde{g}}^{h}+\beta \frac{\widetilde{f}^{h} \widetilde{\widetilde{f}}^{h}}{f^{h^{2}}} d \mathbf{x},
\end{aligned}
$$

where $\mathbf{u}^{h} \in \mathcal{U}^{h}$ is the solution of

$$
\left\langle\nabla \cdot \mathbf{u}^{h}, \nabla \cdot \mathbf{v}^{h}\right\rangle+\left\langle\nabla \times \mathbf{u}^{h}, \nabla \times \mathbf{v}^{h}\right\rangle=\left\langle f^{h}-1, \nabla \cdot \mathbf{v}^{h}\right\rangle+\left\langle g^{h}, \nabla \times \mathbf{v}^{h}\right\rangle,
$$

for all $\mathbf{v}^{h} \in \mathcal{U}^{h}, \phi^{h}$ satisfies (3.8), the first variations $\widetilde{\mathbf{u}}^{h}, \widetilde{\mathbf{u}}^{h} \in \mathcal{U}^{h}$ and $\widetilde{\boldsymbol{\phi}}_{n}^{h}, \widetilde{\boldsymbol{\phi}}_{n}^{h} \in \Phi^{h}$ are solutions of

$$
\begin{gathered}
\left\langle\nabla \cdot \widetilde{\mathbf{u}}^{h}, \nabla \cdot \widetilde{\mathbf{v}}^{h}\right\rangle+\left\langle\nabla \times \widetilde{\mathbf{u}}^{h}, \nabla \times \widetilde{\mathbf{v}}^{h}\right\rangle=\left\langle\widetilde{f}^{h}, \nabla \cdot \widetilde{\mathbf{v}}^{h}\right\rangle+\left\langle\widetilde{g}^{h}, \nabla \times \widetilde{\mathbf{v}}^{h}\right\rangle, \\
\left\langle\nabla \cdot \widetilde{\widetilde{\mathbf{u}}}^{h}, \nabla \cdot \widetilde{\widetilde{\mathbf{v}}}^{h}\right\rangle+\left\langle\nabla \times \widetilde{\widetilde{\mathbf{u}}}^{h}, \nabla \times \widetilde{\widetilde{\mathbf{v}}}^{h}\right\rangle=\left\langle\widetilde{\widetilde{f}}^{h}, \nabla \cdot \widetilde{\widetilde{\mathbf{v}}}^{h}\right\rangle+\left\langle\widetilde{\widetilde{g}}^{h}, \nabla \times \widetilde{\widetilde{\mathbf{v}}}^{h}\right\rangle,
\end{gathered}
$$

for all $\widetilde{\mathbf{v}}^{h}, \widetilde{\mathbf{v}}^{h} \in \mathcal{U}^{h}$,

$$
\left\{\begin{array}{l}
\left\langle\frac{\widetilde{\boldsymbol{\phi}}_{n+1}^{h}-\widetilde{\boldsymbol{\phi}}_{n}^{h}}{\Delta t}-\widetilde{\boldsymbol{\phi}}_{n}^{h} \cdot \nabla_{\boldsymbol{\phi}} \mathbf{u}^{h}\left(\boldsymbol{\phi}_{n}^{h}\right)-\widetilde{\mathbf{u}}^{h}\left(\boldsymbol{\phi}_{n}^{h}\right), \widetilde{\rho}^{h}\right\rangle=0, \quad \forall \widetilde{\rho}^{h} \in \Phi^{h} \\
\widetilde{\boldsymbol{\phi}}_{0}^{h}(\mathbf{x})=\mathbf{0}, \quad \text { in } \Omega,
\end{array}\right.
$$

and

$$
\left\{\begin{array}{l}
\left\langle\frac{\widetilde{\widetilde{\phi}}_{n+1}^{h}-\widetilde{\widetilde{\phi}}_{n}^{h}}{\Delta t}-\widetilde{\widetilde{\phi}}_{n}^{h} \cdot \nabla_{\phi} \mathbf{u}^{h}\left(\phi_{n}^{h}\right)-\widetilde{\mathbf{u}}^{h}\left(\phi_{n}^{h}\right), \widetilde{\widetilde{\rho}}^{h}\right\rangle=0, \quad \forall \widetilde{\widetilde{\rho}}^{h} \in \Phi^{h} \\
\widetilde{\widetilde{\phi}}_{0}^{h}(\mathbf{x})=\mathbf{0}, \quad \text { in } \Omega,
\end{array}\right.
$$


respectively, and the second variations $\widehat{\mathbf{u}}^{h} \in \mathcal{U}^{h}$ and $\widehat{\phi}^{h} \in \Phi$ are solutions of

$$
\left\langle\nabla \cdot \widehat{\mathbf{u}}^{h}, \nabla \cdot \widehat{\mathbf{v}}^{h}\right\rangle+\left\langle\nabla \times \widehat{\mathbf{u}}^{h}, \nabla \times \widehat{\mathbf{v}}^{h}\right\rangle=0, \quad \forall \widehat{\mathbf{v}}^{h} \in \mathcal{U}^{h}
$$

and

$$
\left\{\begin{array}{l}
\left\langle\frac{\widehat{\boldsymbol{\phi}}_{n+1}^{h}-\widehat{\boldsymbol{\phi}}_{n}^{h}}{\Delta t}-\widehat{\boldsymbol{\phi}}_{n}^{h} \cdot \nabla_{\boldsymbol{\phi}} \mathbf{u}^{h}\left(\boldsymbol{\phi}_{n}^{h}\right)-\widetilde{\boldsymbol{\phi}}_{n}^{h} \cdot\left(\widetilde{\widetilde{\boldsymbol{\phi}}}_{n}^{h} \cdot \nabla_{\boldsymbol{\phi}} \nabla_{\boldsymbol{\phi}} \mathbf{u}^{h}\left(\boldsymbol{\phi}_{n}^{h}\right)\right)\right. \\
\left.-\widetilde{\boldsymbol{\phi}}_{n}^{h} \cdot \nabla_{\boldsymbol{\phi}} \widetilde{\widetilde{\mathbf{u}}}^{h}\left(\boldsymbol{\phi}_{n}^{h}\right)-\widetilde{\widetilde{\phi}}_{n}^{h} \cdot \nabla_{\boldsymbol{\phi}} \widetilde{\mathbf{u}}^{h}\left(\boldsymbol{\phi}_{n}^{h}\right)-\widehat{\mathbf{u}}^{h}\left(\boldsymbol{\phi}_{n}^{h}\right), \widehat{\rho}^{h}\right\rangle=0, \quad \forall \widehat{\rho}^{h} \in \Phi^{h} \\
\widehat{\boldsymbol{\phi}}_{0}^{h}(\mathbf{x})=\mathbf{0}, \quad \text { in } \Omega,
\end{array}\right.
$$

respectively. The equation (4.8) yields $\widehat{\mathbf{u}}^{h}=\mathbf{0}$ so that we can omit the last term $\widehat{\mathbf{u}}^{h}\left(\boldsymbol{\phi}_{n}^{h}\right)$ in (4.9).

First we show

$$
c\left(\left\|\widetilde{f}^{h}\right\|^{2}+\left\|\widetilde{g}^{h}\right\|^{2}\right) \leq D^{2} J^{h}\left(\phi_{N}^{h} ; f^{h}, g^{h}\right)\left[\left(\widetilde{f}^{h}, \widetilde{g}^{h}\right),\left(\widetilde{f}^{h}, \widetilde{g}^{h}\right)\right] .
$$

By using the final condition of $\boldsymbol{\psi}_{N}^{h}$ at $t=1$ in (3.55) and the Hölder inequality, the second Frechét derivative of $J^{h}$ becomes

$$
\begin{aligned}
& D^{2} J^{h}\left(\boldsymbol{\phi}_{N}^{h} ; f^{h}, g^{h}\right)\left[\left(\widetilde{f}^{h}, \widetilde{g}^{h}\right),\left(\widetilde{f}^{h}, \widetilde{g}^{h}\right)\right]=\int_{\Omega} \widehat{\boldsymbol{\phi}}_{N}^{h}(\mathbf{x}) \cdot \boldsymbol{\psi}_{N}^{h}(\mathbf{x}) \\
& \quad+\int_{\Omega} \widetilde{\boldsymbol{\phi}}_{N}^{h}(\mathbf{x}) \cdot\left(\widetilde{\boldsymbol{\phi}}_{N}^{h}(\mathbf{x}) \cdot \nabla_{\boldsymbol{\phi}} \nabla_{\boldsymbol{\phi}} \mathbf{T}^{h}\left(\boldsymbol{\phi}_{N}^{h}(x)\right)\right)\left(\mathbf{T}^{h}\left(\boldsymbol{\phi}_{N}^{h}(\mathbf{x})\right)-\mathbf{R}^{h}\right) d \mathbf{x} \\
& \quad+\int_{\Omega}\left(\widetilde{\boldsymbol{\phi}}_{N}^{h}(\mathbf{x}) \cdot \nabla_{\boldsymbol{\phi}} \mathbf{T}^{h}\left(\boldsymbol{\phi}_{N}^{h}(\mathbf{x})\right)\right)^{2} \\
& \quad+\int_{\Omega} \alpha_{f_{0}}\left|\widetilde{f}^{h}\right|^{2}+\alpha_{f_{1}}\left|\nabla \widetilde{f}^{h}\right|^{2}+\alpha_{g_{0}}\left|\widetilde{g}^{h}\right|^{2}+\alpha_{g_{1}}\left|\nabla \widetilde{g}^{h}\right|^{2}+\beta \frac{\left|\widetilde{f}^{h}\right|^{2}}{f^{h^{2}}} d \mathbf{x} .
\end{aligned}
$$

We first choose $\widehat{\rho}^{h}=\boldsymbol{\psi}_{n+1}^{h}$ in (4.9). Then equation (3.55) at $t_{n}$ multiplied by $\widehat{\boldsymbol{\phi}}_{n}^{h}$ :

$$
\widehat{\boldsymbol{\phi}}_{n}^{h} \cdot \boldsymbol{\psi}_{n}^{h}=\widehat{\boldsymbol{\phi}}_{n}^{h} \cdot\left(\boldsymbol{\psi}_{n+1}^{h}+\Delta t \nabla_{\boldsymbol{\phi}} \mathbf{u}^{h}\left(\boldsymbol{\phi}_{n}^{h}\right) \boldsymbol{\psi}_{n+1}^{h}\right)
$$

and $\left(\widehat{\boldsymbol{\phi}}_{n}^{h} \cdot \nabla_{\boldsymbol{\phi}} \mathbf{u}^{h}\left(\boldsymbol{\phi}_{n}^{h}\right)\right) \cdot \boldsymbol{\psi}_{n+1}^{h}=\widehat{\boldsymbol{\phi}}_{n}^{h} \cdot\left(\nabla_{\boldsymbol{\phi}} \mathbf{u}^{h}\left(\boldsymbol{\phi}_{n}^{h}\right) \boldsymbol{\psi}_{n+1}^{h}\right)$ change the equation (4.9) to

$$
\begin{aligned}
& \int_{\Omega} \widehat{\boldsymbol{\phi}}_{n+1}^{h} \cdot \boldsymbol{\psi}_{n+1}^{h} d \mathbf{x}=\int_{\Omega} \widehat{\boldsymbol{\phi}}_{n}^{h} \cdot \boldsymbol{\psi}_{n}^{h} d \mathbf{x} \\
& \quad+\Delta t \int_{\Omega}\left(\widetilde{\boldsymbol{\phi}}_{n}^{h} \cdot\left(\widetilde{\boldsymbol{\phi}}_{n}^{h} \nabla_{\boldsymbol{\phi}} \nabla_{\boldsymbol{\phi}} \mathbf{u}^{h}\left(\boldsymbol{\phi}_{n}^{h}\right)\right)+2 \widetilde{\boldsymbol{\phi}}_{n}^{h} \cdot \nabla_{\boldsymbol{\phi}} \widetilde{\mathbf{u}}^{h}\left(\boldsymbol{\phi}_{n}^{h}\right)\right) \cdot \boldsymbol{\psi}_{n+1}^{h} d \mathbf{x} .
\end{aligned}
$$

Induction, the initial condition $\widehat{\boldsymbol{\phi}}_{0}^{h}=\mathbf{0}$, and the Hölder inequality yield

$$
\begin{aligned}
& \int_{\Omega} \widehat{\boldsymbol{\phi}}_{N}^{h} \cdot \boldsymbol{\psi}_{N}^{h} d \mathbf{x} \\
& =\Delta t \sum_{k=0}^{N} \int_{\Omega}\left(\widetilde{\boldsymbol{\phi}}_{k}^{h} \cdot\left(\widetilde{\boldsymbol{\phi}}_{k}^{h} \nabla_{\boldsymbol{\phi}} \nabla_{\boldsymbol{\phi}} \mathbf{u}^{h}\left(\boldsymbol{\phi}_{k}^{h}\right)\right)+2 \widetilde{\boldsymbol{\phi}}_{k}^{h} \cdot \nabla_{\boldsymbol{\phi}} \widetilde{\mathbf{u}}^{h}\left(\boldsymbol{\phi}_{k}^{h}\right)\right) \cdot \boldsymbol{\psi}_{k+1}^{h} d \mathbf{x} . \\
& \geq-\Delta t \sum_{k=0}^{N}\left\{\left(\int_{\Omega}\left|\nabla_{\boldsymbol{\phi}}^{2} \mathbf{u}^{h}\left(\boldsymbol{\phi}_{k}^{h}\right)\right|^{2} d \mathbf{x}\right)^{\frac{1}{2}}\left(\int_{\Omega}\left|\widetilde{\boldsymbol{\phi}}_{k}^{h}\right|^{8} d \mathbf{x}\right)^{\frac{1}{4}}\left(\int_{\Omega}\left|\boldsymbol{\psi}_{k+1}^{h}\right|^{4} d \mathbf{x}\right)^{\frac{1}{4}}\right. \\
& \left.\quad+\left(\int_{\Omega}\left|\nabla_{\boldsymbol{\phi}} \widetilde{\mathbf{u}}^{h}\left(\boldsymbol{\phi}_{k}^{h}\right)\right|^{2} d \mathbf{x}\right)^{\frac{1}{2}}\left(\int_{\Omega}\left|\widetilde{\boldsymbol{\phi}}_{k}^{h}\right|^{4} d \mathbf{x}\right)^{\frac{1}{4}}\left(\int_{\Omega}\left|\boldsymbol{\psi}_{k+1}^{h}\right|^{4} d \mathbf{x}\right)^{\frac{1}{4}}\right\} .
\end{aligned}
$$


We recall the change of variables (3.60):

$$
\left|\operatorname{det} \nabla \phi_{k}^{h}\right| \geq\left.|2-\Delta t|\left\|\nabla \cdot \mathbf{u}^{h}\right\|_{\infty}\right|^{k}
$$

that implies

$$
\int_{\Omega}\left|\nabla_{\phi}^{2} \mathbf{u}^{h}\left(\phi_{k}^{h}\right)\right|^{2} d \mathbf{x} \leq \gamma^{k}\left\|\nabla^{2} \mathbf{u}^{h}\right\|^{2} \quad \text { and } \quad \int_{\Omega}\left|\nabla_{\phi} \widetilde{\mathbf{u}}^{h}\left(\phi_{k}^{h}\right)\right|^{2} d \mathbf{x} \leq \gamma^{k}\left\|\nabla \widetilde{\mathbf{u}}^{h}\right\|^{2},
$$

where $\gamma=1 /|2-\Delta t|\left|\nabla \cdot \mathbf{u}^{h} \|_{\infty}\right|$.

Now, we consider the term $\left\|\boldsymbol{\psi}_{k+1}^{h}\right\|_{L^{4}(\Omega)}$. In (3.55) at $t=t_{k}$, we multiply by $\left(\boldsymbol{\psi}_{k}^{h}\right)^{3}$ to the equation and integrate it over $\Omega$. Applying the Hölder inequality, the change of variables (4.12), and the inverse inequality (3.11) results in

$$
\begin{aligned}
& \left\|\boldsymbol{\psi}_{k}^{h}\right\|_{L^{4}(\Omega)}^{4} \\
& \leq\left\|\boldsymbol{\psi}_{k+1}^{h}\right\|_{L^{4}(\Omega)}\left\|\boldsymbol{\psi}_{k}^{h}\right\|_{L^{4}(\Omega)}^{3}+\Delta t\left\|\nabla_{\boldsymbol{\phi}} \mathbf{u}^{h}\left(\boldsymbol{\phi}_{k}^{h}\right)\right\|_{L^{2}(\Omega)}\left\|\boldsymbol{\psi}_{k+1}^{h}\right\|_{L^{4}(\Omega)}\left\|\boldsymbol{\psi}_{k}^{h}\right\|_{L^{12}(\Omega)}^{3} \\
& \leq\left\|\boldsymbol{\psi}_{k+1}^{h}\right\|_{L^{4}(\Omega)}\left\|\boldsymbol{\psi}_{k}^{h}\right\|_{L^{4}(\Omega)}^{3}+\Delta t \gamma^{\frac{k}{2}}\left\|\nabla \mathbf{u}^{h}\right\|\left\|\boldsymbol{\psi}_{k+1}^{h}\right\|_{L^{4}(\Omega)} c_{1} h^{-1}\left\|\boldsymbol{\psi}_{k}^{h}\right\|_{L^{4}(\Omega)}^{3} .
\end{aligned}
$$

The above inequality, induction, and the final condition in (3.55) with the change of variables used in (4.13) provide

$$
\begin{aligned}
\left\|\boldsymbol{\psi}_{k}^{h}\right\|_{L^{4}(\Omega)} & \leq\left(1+c_{1} \Delta t h^{-1}\left\|\nabla \mathbf{u}^{h}\right\| \gamma^{\frac{k}{2}}\right)\left\|\boldsymbol{\psi}_{k+1}^{h}\right\|_{L^{4}(\Omega)} \\
\cdots & \leq \prod_{m=k}^{N-1}\left(1+c_{1} \Delta t h^{-1}\left\|\nabla \mathbf{u}^{h}\right\| \gamma^{\frac{m}{2}}\right)\left\|\boldsymbol{\psi}_{N}^{h}\right\|_{L^{4}(\Omega)} \\
& \leq \prod_{m=k}^{N-1}\left(1+c_{1} \Delta t h^{-1}\left\|\nabla \mathbf{u}^{h}\right\| \gamma^{\frac{m}{2}}\right) C_{T R} \gamma^{\frac{N}{4}}\left\|\nabla \mathbf{T}^{h}\right\|_{L^{4}(\Omega)}
\end{aligned}
$$

where $C_{T R} \equiv\left\|\mathbf{T}^{h}\right\|_{\infty}+\left\|\mathbf{R}^{h}\right\|_{\infty}$.

Analogously, for $\left\|\phi_{k}^{h}\right\|_{L^{8}(\Omega)}$, we set $\widetilde{\rho}^{h}=\left(\phi_{k+1}^{h}\right)^{7}$ in (4.6) to obtain

$$
\begin{gathered}
\left\|\widetilde{\boldsymbol{\phi}}_{k+1}^{h}\right\|_{L^{8}(\Omega)}^{8} \leq\left\|\widetilde{\boldsymbol{\phi}}_{k}^{h}\right\|_{L^{8}(\Omega)}\left\|\widetilde{\boldsymbol{\phi}}_{k+1}^{h}\right\|_{L^{8}(\Omega)}^{7} \\
+\Delta t\left(\left\|\nabla_{\boldsymbol{\phi}} \mathbf{u}^{h}\left(\boldsymbol{\phi}_{k}^{h}\right)\right\|\left\|\widetilde{\boldsymbol{\phi}}_{k}^{h}\right\|_{L^{8}(\Omega)}\left\|\widetilde{\boldsymbol{\phi}}_{k+1}^{h}\right\|_{L^{\frac{56}{3}}(\Omega)}^{7}+\left\|\widetilde{\mathbf{u}}^{h}\left(\boldsymbol{\phi}_{k}^{h}\right)\right\|_{L^{8}(\Omega)}\left\|\widetilde{\boldsymbol{\phi}}_{k+1}^{h}\right\|_{L^{8}(\Omega)}^{7}\right) .
\end{gathered}
$$

Applying the inverse inequality $\|\cdot\|_{L^{\frac{56}{3}(\Omega)}} \leq c_{2} h^{-\frac{1}{7}}\|\cdot\|_{L^{8}(\Omega)}$, dividing by $\left\|\widetilde{\boldsymbol{\phi}}_{k+1}^{h}\right\|_{L^{8}(\Omega)}^{7}$ on both sides of the inequality (4.15), the change of variables (4.12), the Sobolev imbedding theorem $\|\cdot\|_{L^{8}(\Omega)} \leq c_{3}\|\cdot\|_{H^{1}(\Omega)}$, induction, and the initial condition $\widetilde{\phi}_{0}^{h}=\mathbf{0}$ result in

$$
\begin{aligned}
\left\|\widetilde{\boldsymbol{\phi}}_{k+1}^{h}\right\|_{L^{8}(\Omega)} & \leq\left(1+c_{2} \Delta t h^{-1}\left\|\nabla_{\phi_{\mathbf{u}}} \mathbf{u}^{h}\left(\boldsymbol{\phi}_{k}^{h}\right)\right\|\right)\left\|\widetilde{\boldsymbol{\phi}}_{k}^{h}\right\|_{L^{8}(\Omega)}+\Delta t\left\|\widetilde{\mathbf{u}}^{h}\left(\boldsymbol{\phi}_{k}^{h}\right)\right\|_{L^{8}(\Omega)} \\
& \leq\left(1+c_{2} \Delta t h^{-1}\left\|\nabla \mathbf{u}^{h}\right\| \gamma^{\frac{k}{2}}\right)\left\|\widetilde{\boldsymbol{\phi}}_{k}^{h}\right\|_{L^{8}(\Omega)}+\gamma^{\frac{k}{8}} \Delta t c_{3}\left\|\widetilde{\mathbf{u}}^{h}\right\|_{1} \\
& \cdots \leq \prod_{m=0}^{k} a_{m}\left\|\widetilde{\boldsymbol{\phi}}_{0}^{h}\right\|_{L^{8}(\Omega)}+\left(b_{k}+\sum_{m=0}^{k-1} b_{m}\left(\prod_{l=m+1}^{k} a_{l}\right)\right)\left\|\widetilde{\mathbf{u}}^{h}\right\|_{1} \\
& =\left(b_{k}+\sum_{m=0}^{k-1} b_{m}\left(\prod_{l=m+1}^{k} a_{l}\right)\right)\left\|\widetilde{\mathbf{u}}^{h}\right\|_{1} \leq\left(\prod_{l=1}^{k} a_{l}\right)\left(\sum_{m=0}^{k} b_{m}\right)\left\|\widetilde{\mathbf{u}}^{h}\right\|_{1},
\end{aligned}
$$


where $a_{l}=1+c_{2} \Delta t h^{-1}\left\|\nabla \mathbf{u}^{h}\right\| \gamma^{\frac{l}{2}}$ and $b_{m}=c_{3} \gamma^{\frac{m}{8}} \Delta t$.

We use a similar process as that in (4.16) to obtain the $L^{4}$-norm bound:

$$
\left\|\widetilde{\boldsymbol{\phi}}_{N}^{h}\right\|_{L^{4}(\Omega)} \leq\left(\prod_{l=1}^{N} p_{l}\right)\left(\sum_{m=0}^{N} q_{m}\right)\left\|\widetilde{\mathbf{u}}^{h}\right\|_{1},
$$

where $p_{k}=1+c_{4} \Delta t h^{-1}\left\|\nabla \mathbf{u}^{h}\right\| \gamma^{\frac{k}{2}}$ and $q_{k}=c_{5} \gamma^{\frac{k}{4}} \Delta t$ with constants $c_{4}$ and $c_{5}$ from $\|\cdot\|_{L^{12}(\Omega)}^{3} \leq c_{4} h^{-1}\|\cdot\|_{L^{4}(\Omega)}^{3}$ and $\|\cdot\|_{L^{4}(\Omega)} \leq c_{5}\|\cdot\|_{1}$, respectively.

Let $\widehat{c}=\max \left\{c_{1}, c_{2}, c_{4}\right\}$. Then, gathering (4.13), (4.14), (4.16), (4.17) and applying them to (4.11) yields

$$
\begin{array}{r}
\int_{\Omega} \widehat{\boldsymbol{\phi}}_{N}^{h} \cdot \boldsymbol{\psi}_{N}^{h} d \mathbf{x} \geq-\Delta t \sum_{k=0}^{N}\left\{\gamma^{\frac{k}{2}}\left\|\nabla^{2} \mathbf{u}^{h}\right\| \cdot A_{k}^{2} B_{k}^{2}\left\|\widetilde{\mathbf{u}}^{h}\right\|_{1}^{2} \cdot A_{k} \gamma^{\frac{N}{4}} C_{T R} c_{5}\left\|\nabla \mathbf{T}^{h}\right\|_{1}\right. \\
\left.+2 \gamma^{\frac{k}{2}}\left\|\nabla \widetilde{\mathbf{u}}^{h}\right\| \cdot A_{k} \bar{B}_{k}\left\|\widetilde{\mathbf{u}}^{h}\right\|_{1} \cdot A_{k} \gamma^{\frac{N}{4}} C_{T R} c_{5}\left\|\nabla \mathbf{T}^{h}\right\|_{1}\right\} \\
\geq-\Delta t \sum_{k=0}^{N}\left\{\gamma^{\frac{k}{2}} \gamma^{\frac{N}{4}}\left\|\widetilde{\mathbf{u}}^{h}\right\|_{1}^{2} C_{0} A_{k}^{2}\left(\left\|\nabla^{2} \mathbf{u}^{h}\right\| A_{k} B_{k}^{2}+2 \bar{B}_{k}\right)\right\}
\end{array}
$$

where $A_{k}=\prod_{l=1}^{k}\left(1+\widehat{c} \Delta t h^{-1}\left\|\nabla \mathbf{u}^{h}\right\| \gamma^{\frac{l}{2}}\right), B_{k}=c_{3} \Delta t \sum_{m=0}^{k} \gamma^{\frac{m}{8}}$, $\bar{B}_{k}=c_{5} \Delta t \sum_{m=0}^{k} \gamma^{\frac{m}{4}}$, and $C_{0}=C_{T R} c_{5}\left\|\nabla \mathbf{T}^{h}\right\|_{1}$.

Suppose we choose $\Delta t$ satisfying

$$
\Delta t \leq \frac{\epsilon^{2}+2 \epsilon-1}{(1+\epsilon)^{2}} \min \left\{\frac{1}{\left\|\nabla \cdot \mathbf{u}^{h}\right\|_{\infty}}, \frac{h}{\widehat{c}\left\|\nabla \mathbf{u}^{h}\right\|}\right\}
$$

for $\epsilon \in\left(\sqrt{2}-1, \frac{\sqrt{5}-1}{2}\right] \sim(0.4142,0.6810]$. Then,

$$
\gamma \leq\left(\frac{1+\epsilon}{2}\right)^{2}<1 \quad \text { and } \quad \widehat{c} \Delta t h^{-1}\left\|\nabla \mathbf{u}^{h}\right\| \leq \frac{\epsilon^{2}+2 \epsilon-1}{(1+\epsilon)^{2}} \leq \frac{1-\epsilon}{1+\epsilon}
$$

Similarly to (3.67), we have $A_{k} \leq \prod_{m=1}^{k}\left(1+\frac{1-\epsilon}{1+\epsilon} \cdot\left(\frac{1+\epsilon}{2}\right)^{m}\right) \leq e$. Thus, we have $A_{K}^{2} \leq e^{2}$ and

$$
\begin{gathered}
\left\|\nabla^{2} \mathbf{u}^{h}\right\| A_{k} B_{k}^{2}+2 \bar{B}_{k} \leq\left\|\nabla^{2} \mathbf{u}^{h}\right\| e \frac{2 c_{3}^{2} \Delta t^{2}}{1-\gamma^{\frac{1}{4}}}+\frac{2 c_{5} \Delta t}{1-\gamma^{\frac{1}{4}}} \\
\leq \frac{2 \Delta t}{1-\gamma^{\frac{1}{4}}}\left(\frac{c_{3}^{2} c_{6} e(1-\epsilon)}{\widehat{c}(1+\epsilon)}+c_{5}\right) \leq \Delta t \frac{4+2 \sqrt{2+2 \epsilon}}{1-\epsilon}\left(\frac{c_{3}^{2} c_{6} e(1-\epsilon)}{\widehat{c}(1+\epsilon)}+c_{5}\right),
\end{gathered}
$$

where the constant $c_{6}$ stems from an inverse inequality $\left\|\nabla^{2} \mathbf{u}^{h}\right\| \leq c_{6} h^{-1}\left\|\nabla \mathbf{u}^{h}\right\|$.

Hence, we have

$$
\int_{\Omega} \widehat{\boldsymbol{\phi}}_{N}^{h} \cdot \boldsymbol{\psi}_{N}^{h} d \mathbf{x} \geq-\Delta t^{2} \bar{K}_{1}\left\|\widetilde{\mathbf{u}}^{h}\right\|_{1}^{2} \sum_{k=0}^{N} \gamma^{\frac{k}{2}} \geq-\Delta t^{2} \bar{K}_{1} \frac{2}{1-\epsilon}\left\|\widetilde{\mathbf{u}}^{h}\right\|_{1}^{2},
$$


where $\bar{K}_{1}=e^{2} \cdot c_{5} C_{T R}\left\|\nabla \mathbf{T}^{h}\right\|_{1}\left(\frac{1+\epsilon}{2}\right)^{\frac{1}{2 \Delta t}} \cdot \frac{4+2 \sqrt{2+2 \epsilon}}{1-\epsilon}\left(\frac{c_{3}^{2} c_{6} e(1-\epsilon)}{\widehat{c}(1+\epsilon)}+c_{5}\right)$ and therefore

$$
\begin{aligned}
& \int_{\Omega} \widehat{\boldsymbol{\phi}}_{N}^{h}(\mathbf{x}) \cdot \boldsymbol{\psi}_{N}^{h}(\mathbf{x})+\widetilde{\boldsymbol{\phi}}_{N}^{h}(\mathbf{x}) \cdot\left(\widetilde{\boldsymbol{\phi}}_{N}^{h}(\mathbf{x}) \cdot \nabla_{\boldsymbol{\phi}}^{2} \mathbf{T}^{h}\left(\boldsymbol{\phi}_{N}^{h}(x)\right)\right)\left(\mathbf{T}^{h}\left(\boldsymbol{\phi}_{N}^{h}(\mathbf{x})\right)-\mathbf{R}^{h}\right) d \mathbf{x} \\
& \quad \geq \int_{\Omega} \widehat{\boldsymbol{\phi}}_{N}^{h}(\mathbf{x}) \cdot \boldsymbol{\psi}_{N}^{h}(\mathbf{x}) d \mathbf{x}-C_{T R}\left\|\widetilde{\boldsymbol{\phi}}_{N}^{h}\right\|_{L^{4}(\Omega)}^{2}\left\|\nabla_{\boldsymbol{\phi}}^{2} \mathbf{T}^{h}\left(\boldsymbol{\phi}_{N}^{h}(\mathbf{x})\right)\right\| \\
& \quad \geq-\Delta t^{2} K\left\|\widetilde{\mathbf{u}}^{h}\right\|_{1}^{2},
\end{aligned}
$$

where $K=K_{1}+K_{2}$ with $K_{1}=2 \bar{K}_{1} /(1-\epsilon)$ and $K_{2}=C_{T R} \cdot e^{2} \cdot \frac{4}{1-\epsilon} \cdot c_{5}^{2} \cdot\left(\frac{1+\epsilon}{2}\right)^{\frac{1}{\Delta t}}$. $\left\|\nabla^{2} \mathbf{T}^{h}\right\|$.

Now, we use the following regularity result for (4.4) to complete the proof of the coercivity of $D^{2} J^{h}$ : there exists a constant $\widetilde{C}$ such that

$$
\left\|\widetilde{\mathbf{u}}^{h}\right\|_{1}^{2} \leq \widetilde{C}\left(\left\|\widetilde{f}^{h}\right\|^{2}+\left\|\widetilde{g}^{h}\right\|^{2}\right)
$$

Therefore, (4.22) with (4.21) yields

$$
\begin{aligned}
& D^{2} J^{h}\left(\boldsymbol{\phi}_{N}^{h} ; f^{h}, g^{h}\right)\left[\left(\widetilde{f}^{h}, \widetilde{g}^{h}\right),\left(\widetilde{f}^{h}, \widetilde{g}^{h}\right)\right] \\
& \geq \int_{\Omega} \widehat{\boldsymbol{\phi}}_{N}^{h}(\mathbf{x}) \cdot \boldsymbol{\psi}_{N}^{h}(\mathbf{x}) d \mathbf{x}-C_{T R_{2}}\left\|\widetilde{\boldsymbol{\phi}}_{N}^{h}\right\|^{2}+\int_{\Omega}\left(\widetilde{\boldsymbol{\phi}}_{N}^{h}(\mathbf{x}) \cdot \nabla_{\boldsymbol{\phi}} \mathbf{T}^{h}\left(\boldsymbol{\phi}_{N}^{h}(\mathbf{x})\right)\right)^{2} d \mathbf{x} \\
& \quad+\alpha_{f_{0}}\left\|\widetilde{f}^{h}\right\|^{2}+\alpha_{f_{1}}\left\|\nabla \widetilde{f}^{h}\right\|^{2}+\alpha_{g_{0}}\left\|\widetilde{g}^{h}\right\|^{2}+\alpha_{g_{1}}\left\|\nabla \widetilde{g}^{h}\right\|^{2}+\beta \frac{\left\|\widetilde{f}^{h}\right\|^{2}}{\left\|f^{h}\right\|_{\infty}^{2}} \\
& \quad\left(\alpha_{f_{0}}+\beta \frac{\left\|\widetilde{f}^{h}\right\|^{2}}{\left\|f^{h}\right\|_{\infty}^{2}}-\Delta t^{2} K \widetilde{C}\right)\left\|\widetilde{f}^{h}\right\|^{2}+\alpha_{f_{1}}\left\|\nabla \widetilde{f}^{h}\right\|^{2} \\
& \quad+\left(\alpha_{g_{0}}-\Delta t^{2} K \widetilde{C}\right)\left\|\widetilde{g}^{h}\right\|^{2}+\alpha_{g_{1}}\left\|\nabla \widetilde{g}^{h}\right\|^{2},
\end{aligned}
$$

where $C_{T R_{2}}=C_{T R}\left\|\nabla_{\phi}^{2} \mathbf{T}\left(\phi_{N}^{h}\right)\right\| \leq C_{T R} \gamma^{\frac{N}{2}}\left\|\nabla^{2} \mathbf{T}\right\| \leq C_{T R}\left(\frac{1+\epsilon}{2}\right)^{\frac{1}{\Delta t}}\left\|\nabla^{2} \mathbf{T}\right\|$ $\leq 2\left(\|\mathbf{T}\|_{2}^{2}+\|\mathbf{R}\|_{2}^{2}\right)\left(\frac{1+\epsilon}{2}\right)^{\frac{1}{\Delta t}}$, if $\Delta t$ is sufficiently small so that it satisfies

$$
\Delta t \leq \min \left\{\frac{\varpi}{\left\|\nabla \cdot \mathbf{u}^{h}\right\|_{\infty}}, \frac{\varpi h}{\widehat{c}\left\|\nabla \mathbf{u}^{h}\right\|}, \sqrt{\frac{\alpha_{f_{0}}+\frac{\beta\left\|\widetilde{f}^{h}\right\|^{2}}{\left\|f^{h}\right\|_{\infty}^{2}}}{K \widetilde{C}}}, \sqrt{\frac{\alpha_{g_{0}}}{K \widetilde{C}}}\right\}
$$

where $\varpi=\left(\epsilon^{2}+2 \epsilon-1\right) /(1+\epsilon)^{2}$. Of course, the constraint on the time step in (4.24) is a sufficient condition, not a necessary condition. Here, $\left(\epsilon^{2}+2 \epsilon-1\right) /(1+\epsilon)^{2}$ and $K=K_{1}+K_{2}$ are monotonely increasing with respect to $\epsilon$ when $\epsilon$ is in $\left(\sqrt{2}-1, \frac{\sqrt{5}-1}{2}\right]$. However, for fixed $\epsilon$, the value of $K$ decreases as $\Delta t \rightarrow 0$ because of the term $\left(\frac{1+\epsilon}{2}\right)^{\frac{1}{\Delta t}}$ in $K$.

Now, we show

$$
D^{2} J^{h}\left(\phi_{N}^{h} ; f^{h}, g^{h}\right)\left[\left(\widetilde{f}^{h}, \widetilde{g}^{h}\right),\left(\widetilde{\widetilde{f}}^{h}, \widetilde{\widetilde{g}}^{h}\right)\right] \leq c\left(\left\|\widetilde{f}^{h}\right\|+\left\|\widetilde{g}^{h}\right\|\right)\left(\left\|\widetilde{\widetilde{f}}^{h}\right\|+\left\|\widetilde{\widetilde{g}}^{h}\right\|\right) .
$$


By the Hölder inequality,

$$
\begin{aligned}
& D^{2} J^{h}\left(\boldsymbol{\phi}_{N}^{h} ; f^{h}, g^{h}\right)\left[\left(\widetilde{f}^{h}, \widetilde{g}^{h}\right),\left(\widetilde{\widetilde{f}}^{h}, \widetilde{\widetilde{g}}^{h}\right)\right] \\
& \leq \int_{\Omega} \widehat{\boldsymbol{\phi}}_{N}^{h} \cdot \boldsymbol{\psi}_{N}^{h} d \mathbf{x}+C_{T R}\left\|\nabla_{\boldsymbol{\phi}}^{2} \mathbf{T}^{h}\left(\boldsymbol{\phi}_{N}^{h}\right)\right\|\left\|\widetilde{\boldsymbol{\phi}}_{N}^{h}\right\|_{L^{4}(\Omega)}\left\|\widetilde{\widetilde{\boldsymbol{\phi}}}_{N}^{h}\right\|_{L^{4}(\Omega)} \\
& \quad+\left\|\nabla_{\boldsymbol{\phi}} \mathbf{T}^{h}\left(\boldsymbol{\phi}_{N}^{h}\right)\right\|_{L^{4}(\Omega)}^{2}\left\|\widetilde{\boldsymbol{\phi}}_{N}^{h}\right\|_{L^{4}(\Omega)}\left\|\widetilde{\widetilde{\phi}}_{N}^{h}\right\|_{L^{4}(\Omega)} \\
& \quad+\left(\alpha_{f_{0}}+\frac{\beta\left\|\widetilde{f}^{h}\right\|^{2}}{\left\|f^{h}\right\|_{\infty}^{2}}+\alpha_{f_{1}}+\alpha_{g_{0}}+\alpha_{g_{1}}\right)\left(\left\|\widetilde{f}^{h}\right\|_{1}+\left\|\widetilde{g}^{h}\right\|_{1}\right)\left(\left\|\widetilde{\widetilde{f}}^{h}\right\|_{1}+\left\|\widetilde{\widetilde{g}}^{h}\right\|_{1}\right) .
\end{aligned}
$$

The following holds by (4.13)-(4.14), (4.16), and (4.17):

$$
\begin{aligned}
& \int_{\Omega} \widehat{\boldsymbol{\phi}}_{N}^{h} \cdot \boldsymbol{\psi}_{N}^{h} d \mathbf{x} \\
& =\Delta t \sum_{k=0}^{N}\left(\widetilde{\boldsymbol{\phi}}_{k}^{h} \cdot\left(\widetilde{\widetilde{\phi}}_{k}^{h} \cdot \nabla_{\boldsymbol{\phi}} \nabla_{\boldsymbol{\phi}} \mathbf{u}^{h}\left(\boldsymbol{\phi}_{k}^{h}\right)\right)+\widetilde{\boldsymbol{\phi}}_{k}^{h} \cdot \nabla_{\boldsymbol{\phi}} \widetilde{\mathbf{u}}^{h}\left(\boldsymbol{\phi}_{k}^{h}\right)+\widetilde{\widetilde{\phi}}_{k}^{h} \cdot \nabla_{\boldsymbol{\phi}} \widetilde{\mathbf{u}}^{h}\left(\boldsymbol{\phi}_{k}^{h}\right)\right) \cdot \boldsymbol{\psi}_{k+1}^{h} \\
& \leq \Delta t^{2} K_{1}\left\|\widetilde{\mathbf{u}}^{h}\right\|_{1}\left\|\widetilde{\mathbf{u}}^{h}\right\|_{1} .
\end{aligned}
$$

Also, the inequality (4.17) and a change of variables yield

$$
\begin{aligned}
\left(C_{T R}\left\|\nabla_{\boldsymbol{\phi}}^{2} \mathbf{T}^{h}\left(\boldsymbol{\phi}_{N}^{h}\right)\right\|\right. & \left.+\left\|\nabla_{\boldsymbol{\phi}} \mathbf{T}^{h}\left(\boldsymbol{\phi}_{N}^{h}\right)\right\|_{L^{4}(\Omega)}^{2}\right)\left\|\widetilde{\boldsymbol{\phi}}_{N}^{h}\right\|_{L^{4}(\Omega)}\left\|\widetilde{\widetilde{\boldsymbol{\phi}}}_{N}^{h}\right\|_{L^{4}(\Omega)} \\
& \leq \Delta t^{2} K_{3}\left\|\widetilde{\mathbf{u}}^{h}\right\|_{1}\left\|\widetilde{\widetilde{\mathbf{u}}}^{h}\right\|_{1}
\end{aligned}
$$

where $K_{3}=\left(\frac{1+\epsilon}{2}\right)^{\frac{1}{\Delta t}}\left(C_{T R}\left\|\nabla^{2} \mathbf{T}^{h}\right\|+c_{5}^{2}\left\|\nabla \mathbf{T}^{h}\right\|_{1}^{2}\right) \frac{4 c_{5}^{2} e^{2}}{1-\epsilon}$. Again, the regularity results (4.22) and $\left\|\widetilde{\mathbf{u}}^{h}\right\|_{1}^{2} \leq c\left(\left\|\widetilde{\widetilde{f}}^{h}\right\|^{2}+\left\|\widetilde{\widetilde{g}}^{h}\right\|^{2}\right)$ with (4.25) and (4.26) imply

$$
D^{2} J^{h}\left(\phi_{N}^{h} ; f^{h}, g^{h}\right)\left[\left(\widetilde{f}^{h}, \widetilde{g}^{h}\right),\left(\widetilde{\widetilde{f}}^{h}, \widetilde{\widetilde{g}}^{h}\right)\right] \leq K_{4}\left(\left\|\widetilde{f}^{h}\right\|+\left\|\widetilde{g}^{h}\right\|\right)\left(\left\|\widetilde{\widetilde{f}}^{h}\right\|+\left\|\widetilde{\widetilde{g}}^{h}\right\|\right),
$$

where $K_{4}=c \widetilde{C} \Delta t^{2}\left(K_{1}+K_{3}\right)+\alpha_{f_{0}}+\frac{\left\|\beta \widetilde{f}^{h}\right\|^{2}}{\left\|f^{h}\right\|_{\infty}^{2}}+\alpha_{f_{1}}+\alpha_{g_{0}}+\alpha_{g_{1}}$.

Hence (4.23) and (4.27) imply (4.2) and thus the gradient algorithm converges if $\left(f^{h}(0), g^{h}(0)\right) \in B$. $\square$

In Section 3, we showed the convergence of the solution of the discrete optimality system (3.53)-(3.57) to the solution of the optimality system (2.4)-(2.8), i.e.,

$$
\left(\mathbf{u}^{h}, \boldsymbol{\phi}_{N}^{h}, \boldsymbol{\psi}_{N}^{h}, \xi^{h}, \eta^{h}, f^{h}, g^{h}\right) \rightarrow(\mathbf{u}, \boldsymbol{\phi}, \boldsymbol{\psi}, \xi, \eta, f, g)
$$

as $h, \Delta t=1 / N \rightarrow 0$. Theorem 4.1 shows that the iterates of the gradient algorithm coverage to the discrete solution, i.e.,

$\left(\mathbf{u}^{h}(m), \boldsymbol{\phi}_{N}^{h}(m), \boldsymbol{\psi}_{N}^{h}(m), \xi^{h}(m), \eta^{h}(m), f^{h}(m), g^{h}(m)\right) \rightarrow\left(\mathbf{u}^{h}, \boldsymbol{\phi}_{N}^{h}, \boldsymbol{\psi}_{N}^{h}, \xi^{h}, \eta^{h}, f^{h}, g^{h}\right)$,

as $m \rightarrow \infty$. Therefore, the approximations from discrete gradient method converges to the solution of the optimal system, i.e.,

$$
\left(\mathbf{u}^{h}(m), \boldsymbol{\phi}_{N}^{h}(m), \boldsymbol{\psi}_{N}^{h}(m), \xi^{h}(m), \eta^{h}(m), f^{h}(m), g^{h}(m)\right) \rightarrow(\mathbf{u}, \boldsymbol{\phi}, \boldsymbol{\psi}, \xi, \eta, f, g),
$$

as $h, \Delta t \rightarrow 0$ and $m \rightarrow \infty$. 
5. Conclusions. In previous work [12], we apply the grid deformation method and the Lagrange multiplier rule to derive an optimality system that can be used to solve the image registration problem, i.e., to find an optimal transformation that minimizes a dissimilarity measure between the mapping of one given image and another given image. As far as we know, this is the first time this approach is used for the image registration problem. In [12], we also provide the results of several computational experiments that illustrate the effectiveness of our approach.

This paper defines finite element discretizations of the optimality system and analyzes the convergence of the approximate solutions. As a bonus, our work provides the first thorough analysis of the grid deformation method introduced in $[3,13,16]$. For practical reasons, we define a gradient method to uncouple the components of the discretized optimality system and prove the convergence of that iterative method.

In future work, we will explore more sophisticated uncoupling strategies for solving the discretized optimality system that should improve on the efficiency of the gradient method. We will also explore the use of multigrid methods to improve the efficiency of the solution of the individual components of the discretized optimality system.

\section{REFERENCES}

[1] Y. Amit. A nonlinear variational problem for image matching. SIAM J. Sci. Comput., 15:207224, 1994.

[2] P. Bochev and M. Gunzburger. Least-squares finite element methods, volume 166 of Applied Mathematical Sciences. Springer, New York, 2009.

[3] P. Bochev, G. Liao, and G. dela Pena. Analysis and computation of adaptive moving grids by deformation. Numer. Methods Partial Differential Equations, 12:489-506, 1996.

[4] S. Brenner and L. R. Scott. The mathematical theory of finite element methods, volume 15 of Texts in Applied Mathematics. Springer-Verlag, New York, second edition, 2002.

[5] Z. Cai, T. Manteuffel, S. McCormick, and J. Ruge. First-order system 1l* (FOSLL*): scalar elliptic partial differential equations. SIAM J. Numer. Anal., 39:1418-1445 (electronic), 2001.

[6] P. Ciarlet. Introduction to numerical linear algebra and optimisation. Cambridge Texts in Applied Mathematics. Cambridge University Press, Cambridge, 1989. With the assistance of Bernadette Miara and Jean-Marie Thomas, Translated from the French by A. Buttigieg.

[7] P. Ciarlet. The finite element method for elliptic problems, volume 40 of Classics in Applied Mathematics. Society for Industrial and Applied Mathematics (SIAM), Philadelphia, PA, 2002. Reprint of the 1978 original [North-Holland, Amsterdam].

[8] B. Fischer and J. Modersitzki. Curvature based image registration. J. Math. Imaging Vision, 18:81-85, 2003. Special issue on imaging science (Boston, MA, 2002).

[9] V. Girault and P.-A. Raviart. Finite element methods for Navier-Stokes equations, volume 5 of Springer Series in Computational Mathematics. Springer-Verlag, Berlin, 1986. Theory and algorithms.

[10] M. Gunzburger. Perspectives in flow control and optimization, volume 5 of Advances in Design and Control. Society for Industrial and Applied Mathematics (SIAM), Philadelphia, PA, 2003.

[11] S. Keeling and W. Ring. Medical image registration and interpolation by optical flow with maximal rigidity. J. Math. Imaging Vision, 23:47-65, 2005.

[12] E. Lee and M. Gunzburger. An optimal control formulation of an image registration problem. submitted.

[13] G. Liao, T.-W. Pan, and J. Su. Numerical grid generator based on Moser's deformation method. Numer. Methods Partial Differential Equations, 10:21-31, 1994.

[14] J. Antoine Maintz and M. Vierge. A survey of medical image registration. Medical Image Analysis, 2:1-36, 1998 .

[15] J. Modersitzki. Numerical methods for image registration. Numerical Mathematics and Scientific Computation. Oxford University Press, New York, 2004. Oxford Science Publications.

[16] W. Semper and G. Liao. A moving grid finite-element method using grid deformation. Numer. Methods Partial Differential Equations, 11(6):603-615, 1995. 